\title{
Extreme HBL behavior of Markarian 501 during 2012 ${ }^{\star}$
}

M. L. Ahnen ${ }^{1}$, S. Ansoldi ${ }^{2,19}$, L. A. Antonelli ${ }^{3}$, C. Arcaro ${ }^{4}$, A. Babić ${ }^{5}$, B. Banerjee ${ }^{6}$, P. Bangale ${ }^{7}$, U. Barres de Almeida $^{7,22}$, J. A. Barrio ${ }^{8}$, J. Becerra González ${ }^{9}$, W. Bednarek ${ }^{10}$, E. Bernardini ${ }^{11,23}$, A. Berti ${ }^{2,24}$, W. Bhattacharyya ${ }^{11}$, O. Blanch ${ }^{12}$, G. Bonnoli ${ }^{13}$, R. Carosi ${ }^{13}$, A. Carosi ${ }^{3}$, A. Chatterjee ${ }^{6}$, S. M. Colak ${ }^{12}$, P. Colin ${ }^{7}$, E. Colombo ${ }^{9}$,

J. L. Contreras ${ }^{8}$, J. Cortina ${ }^{12}$, S. Covino ${ }^{3}$, P. Cumani ${ }^{12}$, P. Da Vela ${ }^{13}$, F. Dazzi ${ }^{3}$, A. De Angelis ${ }^{4}$, B. De Lotto ${ }^{2}$, M. Delfino ${ }^{12,25}$, J. Delgado ${ }^{12}$, F. Di Pierro ${ }^{4}$, M. Doert ${ }^{14}$, A. Domínguez ${ }^{8}$, D. Dominis Prester ${ }^{5}$, M. Doro ${ }^{4}$,

D. Eisenacher Glawion ${ }^{15}$, M. Engelkemeier ${ }^{14}$, V. Fallah Ramazani ${ }^{16}$, A. Fernández-Barral ${ }^{12}$, D. Fidalgo ${ }^{8}$, M. V. Fonseca ${ }^{8}$, L. Font ${ }^{17}$, C. Fruck ${ }^{7}$, D. Galindo ${ }^{18}$, R. J. García López ${ }^{9}$, M. Garczarczyk ${ }^{11}$, M. Gaug ${ }^{17}$, P. Giammaria ${ }^{3}$, N. Godinović ${ }^{5}$, D. Gora ${ }^{11}$, D. Guberman ${ }^{12}$, D. Hadasch ${ }^{19}$, A. Hahn ${ }^{7}$, T. Hassan ${ }^{12}$, M. Hayashida ${ }^{19}$,

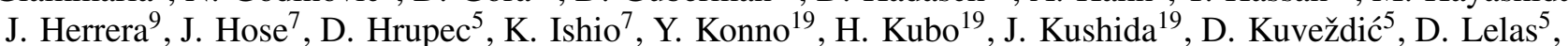
E. Lindfors ${ }^{16}$, S. Lombardi ${ }^{3}$, F. Longo ${ }^{2,24}$, M. López ${ }^{8}$, C. Maggio ${ }^{17}$, P. Majumdar ${ }^{6}$, M. Makariev ${ }^{20}$, G. Maneva ${ }^{20}$, M. Manganaro ${ }^{9}$, L. Maraschi ${ }^{3}$, M. Mariotti ${ }^{4}$, M. Martínez ${ }^{12}$, D. Mazin ${ }^{7,19}$, U. Menzel $^{7}$, M. Minev $^{20}$, J. M. Miranda ${ }^{13}$, R. Mirzoyan ${ }^{7}$, A. Moralejo ${ }^{12}$, V. Moreno ${ }^{17}$, E. Moretti ${ }^{7}$, T. Nagayoshi ${ }^{19}$, V. Neustroev ${ }^{16}$, A. Niedzwiecki $^{10}$,

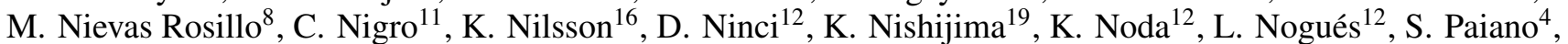
J. Palacio ${ }^{12}$, D. Paneque ${ }^{7, \star \star}$, R. Paoletti ${ }^{13}$, J. M. Paredes ${ }^{18}$, G. Pedaletti ${ }^{11}$, M. Peresano ${ }^{2}$, L. Perri ${ }^{3}$, M. Persic ${ }^{2,26}$, P. G. Prada Moroni ${ }^{21}$, E. Prandini ${ }^{4}$, I. Puljak ${ }^{5}$, J. R. Garcia ${ }^{7}$, I. Reichardt ${ }^{4}$, M. Ribó ${ }^{18}$, J. Rico ${ }^{12}$, C. Righi ${ }^{3}$,

A. Rugliancich ${ }^{13}$, T. Saito ${ }^{19}$, K. Satalecka ${ }^{11}$, S. Schroeder ${ }^{14}$, T. Schweizer ${ }^{7}$, S. N. Shore ${ }^{21}$, J. Sitarek ${ }^{10}$, I. Śnidarić ${ }^{5}$,

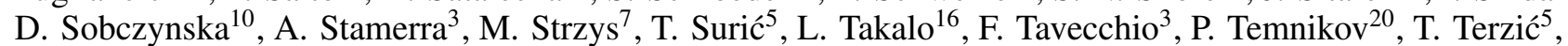

M. Teshima ${ }^{7}, 19$, N. Torres-Albà ${ }^{18}$, A. Treves ${ }^{2}$, S. Tsujimoto ${ }^{19}$, G. Vanzo ${ }^{9}$, M. Vazquez Acosta ${ }^{9}$, I. Vovk $^{7}$,

$$
\text { J. E. Ward }{ }^{12} \text {, M. Will }{ }^{7} \text {, D. Zarićs }{ }^{5}
$$

(MAGIC Collaboration)

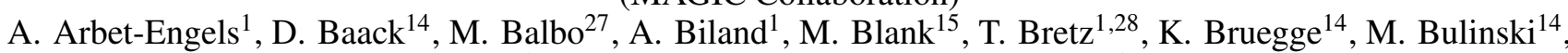
J. Buss ${ }^{14}$, A. Dmytriiev ${ }^{27}$, D. Dorner ${ }^{15}$, S. Einecke ${ }^{14}$, D. Elsaesser ${ }^{14}$, T. Herbst ${ }^{15}$, D. Hildebrand ${ }^{1}$, L. Kortmann ${ }^{14}$, L. Linhoff ${ }^{14}$, M. Mahlke ${ }^{1,28}$, K. Mannheim ${ }^{15}$, S. A. Mueller ${ }^{1}$, D. Neise ${ }^{1}$, A. Neronov $^{27}$, M. Noethe ${ }^{14}$, J. Oberkirch ${ }^{14}$, A. Paravac ${ }^{15}$, W. Rhode ${ }^{14}$, B. Schleicher ${ }^{15}$, F. Schulz ${ }^{14}$, K. Sedlaczek ${ }^{14}$, A. Shukla ${ }^{15}$,, V. Sliusar ${ }^{27}$, R. Walter ${ }^{27}$, (FACT Collaboration)

A. Archer ${ }^{29}$, W. Benbow ${ }^{30}$, R. Bird ${ }^{31}$, R. Brose ${ }^{32,11}$, J. H. Buckley ${ }^{29}$, V. Bugaev ${ }^{29}$, J. L. Christiansen ${ }^{33}$, W. Cui ${ }^{34,35}$, M. K. Daniel ${ }^{30}$, A. Falcone ${ }^{36}$, Q. Feng ${ }^{37}$, J. P. Finley ${ }^{34}$, G. H. Gillanders ${ }^{38}$, O. Gueta ${ }^{11}$, D. Hanna ${ }^{37}$, O. Hervet ${ }^{39}$, J. Holder ${ }^{40}$, G. Hughes ${ }^{1,54, \star \star}$, M. Hütten ${ }^{11}$, T. B. Humensky ${ }^{41}$, C. A. Johnson ${ }^{39}$, P. Kaaret ${ }^{42}$, P. Kar ${ }^{43}$,

N. Kelley-Hoskins ${ }^{11}$, M. Kertzman ${ }^{44}$, D. Kieda ${ }^{43}$, M. Krause ${ }^{11}$, F. Krennrich ${ }^{45}$, S. Kumar ${ }^{37}$, M. J. Lang ${ }^{38}$, T. T. Y. Lin $^{37}$, G. Maier ${ }^{11}$, S. McArthur ${ }^{34}$, P. Moriarty ${ }^{38}$, R. Mukherjee ${ }^{46}$, S. O'Brien ${ }^{47}$, R. A. Ong ${ }^{31}$, A. N. Otte ${ }^{48}$, N. Park ${ }^{49}$, A. Petrashyk ${ }^{41}$, A. Pichel ${ }^{50}$, M. Pohl ${ }^{32,11}$, J. Quinn ${ }^{47}$, K. Ragan ${ }^{37}$, P. T. Reynolds ${ }^{51}$, G. T. Richards ${ }^{40}$,

E. Roache ${ }^{30}$, A. C. Rovero ${ }^{50}$, C. Rulten ${ }^{52}$, I. Sadeh ${ }^{11}$, M. Santander ${ }^{53}$, G. H. Sembroski ${ }^{34}$, K. Shahinyan ${ }^{52}$,

I. Sushch ${ }^{11}$, J. Tyler ${ }^{37}$, S. P. Wakely ${ }^{49}$, A. Weinstein ${ }^{45}$, R. M. Wells ${ }^{45}$, P. Wilcox ${ }^{42}$, A. Wilhel ${ }^{32,11}$, D. A. Williams ${ }^{39}$, T. J Williamson ${ }^{40}$, B. Zitzer ${ }^{37}$,

(VERITAS Collaboration)

M. Perri ${ }^{55,56}$, F. Verrecchia ${ }^{55,56}$, C. Leto ${ }^{55,56}$, M. Villata ${ }^{57}$, C. M. Raiteri ${ }^{57}$, S. G. Jorstad ${ }^{58,82}$, V. M. Larionov $^{58,59}$, D. A. Blinov ${ }^{58,60,61}$, T. S. Grishina ${ }^{58}$, E. N. Kopatskaya ${ }^{58}$, E. G. Larionova ${ }^{58}$, A. A. Nikiforova ${ }^{58,59}$, D. A. Morozova ${ }^{58}$, Yu. V. Troitskaya ${ }^{58}$, I. S. Troitsky ${ }^{58}$, O. M. Kurtanidze ${ }^{62,63,64}$, M. G. Nikolashvili ${ }^{62}$, S. O. Kurtanidze ${ }^{62}$, G. N. Kimeridze ${ }^{62}$, R. A. Chigladze ${ }^{62}$, A. Strigachev ${ }^{65}$, A. C. Sadun $^{66}$, J. W. Moody ${ }^{67}$, W. P. Chen ${ }^{68}$, H. C. Lin $^{68}$, J. A. Acosta-Pulido ${ }^{9}$, M. J. Arévalo 9 , M. I. Carnerero ${ }^{57}$, P. A. González-Morales ${ }^{9}$, A. Manilla-Robles ${ }^{69}$, H. Jermak ${ }^{70}$, I. Steele ${ }^{70}$, C. Mundell ${ }^{70}$, E. Benítez ${ }^{71}$, D. Hiriart ${ }^{72}$, P. S. Smith ${ }^{73}$, W. Max-Moerbeck ${ }^{74}$, A. C. S. Readhead ${ }^{75}$, J. L. Richards ${ }^{34}$, T. Hovatta ${ }^{76}$, A. Lähteenmäki ${ }^{77,78}$, M. Tornikoski ${ }^{77}$, J. Tammi ${ }^{77}$, M. Georganopoulos ${ }^{79,80}$ and M. G. Baring ${ }^{81}$

(Affiliations can be found after the references)

Received 23 June 2018 / Accepted 3 August 2018

\footnotetext{
* The multi-instrument light curves from Fig. 1, and the broadband SED plots from Figs. 5-7 are only available at the CDS via anonymous ftp to http://cdsarc.u-strasbg.fr (ftp://130.79.128.5) or via http://cdsarc.u-strasbg.fr/viz-bin/qcat?J/A+A/620/A181

$\star \star$ Corresponding authors: G. Hughes (e-mail: gareth.hughes@cfa.harvard.edu), D. Paneque (e-mail: dpaneque@mppmu .mpg.de), A. Shukla (e-mail: amit.shukla@astro.uni-wuerzburg.de).
} 


\section{ABSTRACT}

Aims. We aim to characterize the multiwavelength emission from Markarian 501 (Mrk 501), quantify the energy-dependent variability, study the potential multiband correlations, and describe the temporal evolution of the broadband emission within leptonic theoretical scenarios.

Methods. We organized a multiwavelength campaign to take place between March and July of 2012. Excellent temporal coverage was obtained with more than 25 instruments, including the MAGIC, FACT and VERITAS Cherenkov telescopes, the instruments on board the Swift and Fermi spacecraft, and the telescopes operated by the GASP-WEBT collaboration.

Results. Mrk 501 showed a very high energy (VHE) gamma-ray flux above $0.2 \mathrm{TeV}$ of $\sim 0.5$ times the Crab Nebula flux (CU) for most of the campaign. The highest activity occurred on 2012 June 9 , when the VHE flux was $\sim 3 \mathrm{CU}$, and the peak of the high-energy spectral component was found to be at $\sim 2 \mathrm{TeV}$. Both the X-ray and VHE gamma-ray spectral slopes were measured to be extremely hard, with spectral indices $<2$ during most of the observing campaign, regardless of the X-ray and VHE flux. This study reports the hardest Mrk 501 VHE spectra measured to date. The fractional variability was found to increase with energy, with the highest variability occurring at VHE. Using the complete data set, we found correlation between the X-ray and VHE bands; however, if the June 9 flare is excluded, the correlation disappears (significance $<3 \sigma$ ) despite the existence of substantial variability in the X-ray and VHE bands throughout the campaign.

Conclusions. The unprecedentedly hard X-ray and VHE spectra measured imply that their low- and high-energy components peaked above $5 \mathrm{keV}$ and $0.5 \mathrm{TeV}$, respectively, during a large fraction of the observing campaign, and hence that Mrk 501 behaved like an extreme high-frequencypeaked blazar (EHBL) throughout the 2012 observing season. This suggests that being an EHBL may not be a permanent characteristic of a blazar, but rather a state which may change over time. The data set acquired shows that the broadband spectral energy distribution (SED) of Mrk 501, and its transient evolution, is very complex, requiring, within the framework of synchrotron self-Compton (SSC) models, various emission regions for a satisfactory description. Nevertheless the one-zone SSC scenario can successfully describe the segments of the SED where most energy is emitted, with a significant correlation between the electron energy density and the VHE gamma-ray activity, suggesting that most of the variability may be explained by the injection of high-energy electrons. The one-zone SSC scenario used reproduces the behavior seen between the measured $\mathrm{X}$-ray and VHE gamma-ray fluxes, and predicts that the correlation becomes stronger with increasing energy of the X-rays.

Key words. astroparticle physics - acceleration of particles - radiation mechanisms: non-thermal - BL Lacertae objects: general BL Lacertae objects: individual: Mrk501

\section{Introduction}

The galaxy Markarian 501 (Mrk 501; $z=0.034$ ) was first cataloged, along with Markarian 421, in an ultra-violet survey (Markaryan \& Lipovetskii 1972). At very high energies (VHE; $E>100 \mathrm{GeV}$ ) it was first detected by the pioneering Whipple imaging atmospheric-Cherenkov telescope (IACT, Quinn et al. 1996).

Mrk 501 is a BL Lacertae (BL Lac) object, a member of the blazar subclass of active galactic nuclei (AGN), the most common source class in the extragalactic VHE cata$\log ^{1}$. Since the discovery of Mrk 501's VHE emission, it has been extensively studied across all wavelengths. The spectral energy distribution (SED) shows the two characteristic broad peaks, the low-frequency peak from radio to X-ray and the high-frequency peak from X-ray to very high energies. The first peak is thought to originate from synchrotron emission. The second either from inverse-Compton scattering of electrons from the lower-energy component (Marscher \& Gear 1985; Maraschi et al. 1992; Dermer et al. 1992; Sikora et al. 1994) or from the acceleration of hadrons which produce synchrotron emission or interact to produce pions and, in turn, gamma rays (Mannheim 1993; Aharonian 2000; Pohl \& Schlickeiser 2000).

Whilst the typical flux of Mrk 501, above $1 \mathrm{TeV}$, in a nonflaring state, is about one-third that of the Crab Nebula (Crab units; CU $)^{2}$, it has shown extraordinary flaring activity, the first notable examples occuring in 1997 (Catanese et al. 1997; DjannatiAtai et al. 1999; Quinn et al. 1999). Another such flaring episode in the same year (Aharonian et al. 1999) showed the flux above $2 \mathrm{TeV}$ ranged from a fraction of $1 \mathrm{CU}$ to $10 \mathrm{CU}$, with an average of $3 \mathrm{CU}$, and the doubling timescale was found to be as short as $15 \mathrm{TeV}$. In the same period the BeppoSAX Xray satellite reported a hundredfold increase in the energy of the synchrotron peak in coincidence with a hardening of the spectrum. Mrk 501 is an excellent object with which to study blazar phenomena because it is bright and nearby, which permits significant

1 http://tevcat.uchicago.edu

2 In this study we use the Crab Nebula VHE emission reported in Aleksić et al. (2016). The photon flux of the Crab Nebula above $1 \mathrm{TeV}$ is $2 \times 10^{-11} \mathrm{~cm}^{-2} \mathrm{~s}^{-1}$. detections in relatively short observing times in essentially all energy bands. Therefore, the absorption of gamma rays in the extragalactic background light (EBL, Dwek \& Krennrich 2013; Aharonian et al. 2007b; Bonnoli et al. 2015), although not negligible, plays a relatively small role below a few TeV. The flux attenuation factor, $\exp (-\tau)$, at a photon energy of $5 \mathrm{TeV}$ is smaller than 0.5 (for $z=0.034$ ) for most EBL models (Franceschini et al. 2008; Domínguez et al. 2011; Gilmore et al. 2012). In 2008, an extensive multi-instrument program was organized in order to perform an objective (unbiased by flaring states) and detailed study of the temporal evolution, over many years, of the broadband emission of Mrk 501 (see e.g. Abdo et al. 2011; Aleksić et al. 2015a; Furniss et al. 2015; Ahnen et al. 2017).

Here, we report on one of those campaigns, that took place in 2012 and serendipitously observed the largest flare since 1997. This paper is organized as follows: in Sect. 2 the experiments that took part in the campaign are described along with their data analysis. Section 3 describes the multiwavelength light curves from these instruments and is followed by Sects. 4 and 5 , in which the multiband variability and related correlations are characterized. Section 6 characterizes the broadband SED within a standard leptonic scenario, and in Sect. 7, we discuss the implications of the osbservational results reported in this paper. Finally, in Sect. 8, we make some concluding remarks.

\section{Participating instruments}

\subsection{MAGIC}

The Major Atmospheric Gamma-ray Imaging Cherenkov Telescopes (MAGIC) comprise two telescopes located at the Observatorio del Roque de Los Muchachos, La Palma, Canary Islands, Spain $\left(2.2 \mathrm{~km}\right.$ a.s.1., $\left.28^{\circ} 45^{\prime} \mathrm{N} 17^{\circ} 54^{\prime} \mathrm{W}\right)$. Both telescopes are $17 \mathrm{~m}$ in diameter and have a parabolic dish. The system is able to detect air showers initiated by gamma rays in the energy range from $\sim 50 \mathrm{GeV}$ to $\sim 50 \mathrm{TeV}$.

During 2011 and 2012 the readout systems of both telescopes were upgraded, and the camera of MAGIC-I (operational since 2003) was replaced, increasing the density of pixels. 
This resulted in a telescope performance enabling a detection of a $\sim 0.7 \%$ Crab Nebula-like source within $50 \mathrm{~h}$, or a $5 \%$ Crablike flux in $1 \mathrm{~h}$ of observation. The systematic uncertainties in the spectral measurements for a Crab-like point source were estimated to be $11 \%$ in the normalization factor (at $\sim 200 \mathrm{GeV}$ ) and 0.15 in the power-law slope. The systematic uncertainty in the absolute energy determination is estimated to be $15 \%$. Further details about the performance of the MAGIC telescopes after the hardware upgrade in 2011-2012 can be found in Aleksić et al. (2016). The data were analyzed using MARS, the standard analysis package of MAGIC (Zanin et al. 2013; Aleksić et al. 2016). The data from March and April 2012 were taken in stereo mode, whilst the data taken in May and June 2012 were taken with MAGIC-II operating as a single telescope due to a technical issue which precluded the operation of MAGIC-I.

\subsection{VERITAS}

The VERITAS experiment (Very Energetic Radiation Imaging Telescope Array System) is an array of IACTs located at the Fred Lawrence Whipple Observatory in southern Arizona $(1.3 \mathrm{~km}$ a.s.1., N 31 $40^{\prime}$, W $\left.110^{\circ} 57^{\prime}\right)$. It consists of four Davies-Cottontype telescopes.

Full array operations began in September 2007. Each telescope has a focal length and dish diameter of $12 \mathrm{~m}$. The total effective mirror area is $106 \mathrm{~m}^{2}$ and the camera of each telescope is made up of 499 photomultiplier tubes (PMTs). A single pixel has a field of view of $0.15^{\circ}$. The system operates in the energy range from $\sim 100 \mathrm{GeV}$ to $\sim 50 \mathrm{TeV}$.

VERITAS has also undergone several upgrades. In 2009 one of the telescopes was moved in order to make the array more symmetric. During the summer of 2012 the VERITAS cameras were upgraded by replacing all of the photo-multiplier tubes (D. B. Kieda for the VERITAS Collaboration 2013). For more details on the VERITAS instrument see Holder et al. (2008).

The performance of VERITAS is characterized by a sensitivity of $\sim 1 \%$ of the Crab nebula flux to detect (at $5 \sigma$ ) a point-like source in $25 \mathrm{~h}$ of observation, which is equivalent to detecting (at $5 \sigma$ ) a $\sim 5 \%$ Crab flux in $1 \mathrm{~h}$. The uncertainty on the VERITAS energy calibration is approximately $20 \%$. The systematic uncertainty on reconstructed spectral indices is estimated at \pm 0.2 , independent of the source spectral index, according to studies of Madhavan (2013). Further details about the performance of VERITAS can be found on the VERITAS website $^{3}$.

\subsection{FACT}

The First G-APD Cherenkov Telescope (FACT) is the first Cherenkov telescope to use silicon photomultipliers (SiPM/ G-APD) as photodetectors. As such, the camera consists of 1440 G-APD sensors, each with a field of view of $0.11^{\circ}$ providing a total field of view of $4.5^{\circ}$. FACT is located next to the MAGIC telescopes at the Observatorio del Roque de Los Muchachos. The telescope makes use of the old HEGRA CT3 (Mirzoyan 1998) mount, and has a focal length of $5 \mathrm{~m}$ and an effective dish diameter of $\sim 3 \mathrm{~m}$. The telescope operates in the energy range from $\sim 0.8 \mathrm{TeV}$ to $\sim 50 \mathrm{TeV}$. For more details about the design and experimental setup see Anderhub et al. (2013).

Since 2012, FACT has been continuously monitoring known TeV blazars, including Mrk 501 and Mrk 421. FACT provides a dense sampling rate by focusing on a subset of sources and the

\footnotetext{
http://veritas.sao.arizona.edu/specifications
}

ability of the instrument to operate safely during nights of bright ambient light. The data are analyzed and processed immediately, and results are available publicly online ${ }^{4}$ within minutes of the observation (Dorner et al. 2013, 2015; Bretz et al. 2014).

\subsection{Fermi-LAT}

The Large Area Telescope (LAT, Atwood et al. 2009) is a pair-conversion telescope (Fermi Gamma-ray Space Telescope) operating in the energy range from $\sim 30 \mathrm{meV}$ to $>\mathrm{TeV}$. Fermi scans the sky continuously, completing one scan every $3 \mathrm{~h}$. The Fermi-LAT data presented in this paper cover the period from 2011 December 29 (MJD 55924) to 2012 August 13 (MJD 56152). The data were analyzed using the standard Fermi analysis software tools (version v10r1p1), using the P8R2_SOURCE_V6 response function. Events with energy above $0.2 \mathrm{GeV}$ and coming from a $10^{\circ}$ region of interest (ROI) around Mrk 501 were selected, with a $100^{\circ}$ zenith-angle cut to avoid contamination from the Earth's limb. Two background templates were used to model the diffuse Galactic and isotropic extragalactic background, gll_iem_v06.fits and iso_P8R2_SOURCE_V6_v06.txt, respectively ${ }^{5}$. All point sources in the third Fermi-LAT source catalog (3FGL, Acero et al. 2015) located in the $10^{\circ} \mathrm{ROI}$ and an additional surrounding $5^{\circ}$-wide annulus were included in the model. In the unbinned likelihood fit, the spectral parameters were set to the values from the $3 \mathrm{FGL}$, while the normalization parameters of the nine sources within the ROI identified as variable were left free. The normalisation of the diffuse components, as well as the the model parameters related to Mrk 501 were also left free.

Because of the moderate sensitivity of Fermi-LAT to detect Mrk 501 (especially when the source is not flaring), we performed the unbinned likelihood analysis on one-week time intervals for determining the light curves in the two energy bands $0.2-2 \mathrm{GeV}$ and $>2 \mathrm{GeV}$ reported in Sect. 3. In both cases we fixed the PL index to 1.75, as was done in Ahnen et al. (2017). On the other hand, in order to increase the simultaneity with the VHE data, we used 3-day time intervals (centered at the night of the VHE observations) for most of the unbinned likelihood spectral analyses reported in Sect. 6. For those spectral analyses, we performed first the PL fit in the range from $0.2 \mathrm{GeV}$ to $300 \mathrm{GeV}$ (see spectral results in Table B.3). Then, we performed the unbinned likelihood analysis in three energy bins (split equally in $\log$ space from $0.2 \mathrm{GeV}$ to $300 \mathrm{GeV}$ ) where the PL index was fixed to the value retrieved from the spectral fit to the full energy range. Flux upper limits at $95 \%$ confidence level were calculated whenever the test statistic (TS) value ${ }^{6}$ for the source was below $4^{7}$.

\subsection{Swift}

The study reported in this paper makes use of the three instruments on board the Neil Gehrels Swift Gamma-ray Burst Observatory (Gehrels et al. 2004); namely the Burst Alert

\footnotetext{
4 http://fact-project.org/monitoring/

5 http://fermi.gsfc.nasa.gov/ssc/data/access/lat/ BackgroundModels.html

6 The TS value quantifies the probability of having a point gamma-ray source at the location specified. It is roughly the square of the significance value (Mattox et al. 1996).

7 A TS value of 4 corresponds to a $\sim 2 \sigma$ flux measurement, which is a commonly used threshold for flux measurements of known sources.
} 
Telescope (BAT, Markwardt et al. 2005), the X-ray Telescope (XRT, Burrows et al. 2005) and the Ultraviolet/Optical Telescope (UVOT, Roming et al. 2005).

The $15-50 \mathrm{keV}$ hard X-ray fluxes from BAT were retrieved from the transient monitor results provided by the Swift/BAT team (Krimm et al. 2013) ${ }^{8}$, where we made a weighted average of all the observations performed within temporal bins of five days. The BAT count rates are converted to energy flux using that 0.00022 counts cm $\mathrm{cm}^{-2} \mathrm{~s}^{-1}$ corresponds to $1.26 \times 10^{-11} \mathrm{erg} \mathrm{cm}^{-2} \mathrm{~s}^{-1}$ (Krimm et al. 2013). This conversion is strictly correct only for sources with the Crab Nebula spectral index in the BAT energy domain $(\Gamma=2.1)$, but the systematic error for sources with different indices is small and often negligible in comparison with the statistical uncertainties, as reported in Krimm et al. (2013).

The XRT and UVOT data come from dedicated observations organized and performed within the framework of the planned extensive multi-instrument campaign. In this study we consider the 52 Mrk 501 observations performed between 2012 February 2 (MJD 55959) and 2012 July 30 (MJD 56138). All observations were carried out in the Windowed Timing (WT) readout mode, with an average exposure of $0.9 \mathrm{ks}$. The data were processed using the XRTDAS software package (v.2.9.3), which was developed by the ASI Science Data Center and released by HEASARC in the HEASoft package (v.6.15.1). The data are calibrated and cleaned with standard filtering criteria using the xrtpipeline task and calibration files available from the Swift/XRT CALDB (version 20140120). For the spectral analysis, events are selected within a 20-pixel ( 46 arcsec) radius, which contains $90 \%$ of the point-spread function (PSF). The background was estimated from a nearby circular region with a radius of 40 pixels. Corrections for the PSF and CCD defects are applied from response files generated using the xrtmkarf task and the cumulative exposure map. Before the spectra are fitted the $0.3-10 \mathrm{keV}$ data are binned to ensure that there are at least 20 counts in each energy bin. The spectra are then corrected for absorption with a neutral-hydrogen column density fixed to the Galactic $21 \mathrm{~cm}$ value in the direction of Mrk 501, namely $1.55 \times 10^{20} \mathrm{~cm}^{-2}$ (Kalberla et al. 2005).

Swift/UVOT made between 31 and 52 measurements, depending on the filter used. The data telemetry volume was reduced using the image mode, where the photon timing information is discarded and the image is directly accumulated onboard. In this paper we considered UVOT image data taken within the same observations acquired by XRT. Here we use the UV lenticular filters, W1, M2 and W2, which are the ones that are not affected by the strong flux of the host galaxy. We evaluated the photometry of the source according to the recipe in Poole et al. (2008), extracting source counts with an aperture of 5 arcsec radius and an annular background aperture with inner and outer radii of 20 arcsec and 30 arcsec. The count rates were converted to fluxes using the updated calibrations (Breeveld et al. 2011). Flux values were then corrected for mean Galactic extinction using an $E(B-V)$ value of 0.017 (Schlafly \& Finkbeiner 2011) for the UVOT filter effective wavelength and the mean Galactic interstellar extinction curve in Fitzpatrick (1999).

\subsection{Optical instruments}

Optical data in the $R$ band were provided by various telescopes around the world, including the ones from the GASP-WEBT

See http://swift.gsfc.nasa.gov/results/transients/ program (Villata et al. 2008, 2009). In this paper we report observations performed in the $R$ band from the following observatories: Crimean Astrophysical Observatory, St. Petersburg,

Sierra de San Pedro Màrtir, Roque de los Muchachos (KVA), Teide (IAC80), Lulin (SLT), Rozhen $(60 \mathrm{~cm})$, Abastumani $(70 \mathrm{~cm})$, Skinakas, the robotic telescope network AAVSOnet, ROVOR and iTelescopes. The calibration was performed using the stars reported by Villata et al. (1998), the Galactic extinction was corrected using the coefficients given in Schlafly \& Finkbeiner (2011), and the flux from the host galaxy in the $R$ band was estimated using Nilsson et al. (2007) for the apertures of 5 arcsec and 7.5 arcsec used by the various instruments. The reported fluxes include instrument-specific offsets of a few mJy, owing to the different filter spectral responses and analysis procedures of the various optical data sets (e.g. for signal and background extraction) in combination with the strong host-galaxy contribution, which is about $2 / 3$ of the total flux measured in the $R$ band. The offsets applied are the following ones: Abastumani $=3.0 \mathrm{mJy}$; SanPedroMartir $=-1.8 \mathrm{mJy}$; Teide $=2.1 \mathrm{mJy}$; Rozhen $=3.9 \mathrm{mJy}$; Skinakas $=0.8 \mathrm{mJy}$; AAVSOnet $=-3.8 \mathrm{mJy}$; iTelescopes $=-2.5 \mathrm{mJy}$; ROVOR = $-2.7 \mathrm{mJy}$. These offsets were determined using several of the GASP-WEBT instruments as reference, and scaling the other instruments (using simultaneous observations) to match them. Additionally, a point-wise fluctuation of $0.2 \mathrm{mJy}(\sim 0.01 \mathrm{mag})$ was added in quadrature to the statistical errors in order to account for potential day-to-day differences for observations with the same instrument.

We also report on polarization measurements from five facilities: Lowell Observatory (Perkins telescope), St. Petersburg (LX-200), Crimean (AZT-8+ST7), Steward Observatory (2.3 m Bok and $1.54 \mathrm{~m}$ Kuiper telescopes) and Roque de los Muchachos (Liverpool telescope). All polarization measurements were obtained from $R$ band imaging polarimetry, except for the measurements from Steward Observatory, which are derived from spectropolarimetry between $4000 \AA$ and $7550 \AA$ with a resolution of $\sim 15 \AA$. The reported values are constructed from the median $Q / I$ and $U / I$ in the 5000-7000 $\AA$ band. The effective wavelength of this bandpass is similar to the Kron-Cousins $R$ band. The wavelength dependence in the polarization of Mrk 501 seen in the spectro-polarimetry is small and does not significantly affect the variability analysis of the various instruments presented here, as can be deduced from the good agreement between all the instruments shown in the bottom panels of Fig. 1. The details related to the observations and analysis of the polarization data are reported by Larionov et al. (2008), Smith et al. (2009), Jorstad et al. (2010), and Jermak et al. (2016).

\subsection{Radio observations}

We report here radio observations from telescopes at the Metsähovi Radio Observatory and the Owens Valley Radio Observatory (OVRO). The $14 \mathrm{~m}$ Metsähovi Radio telescope operates at $37 \mathrm{GHz}$ and the OVRO at $15 \mathrm{GHz}$. Details of the observation strategies can be found in Teräsranta et al. (1998) and Richards et al. (2011). For both instruments Mrk 501 is a point source, and therefore the measurements represent an integration of the full source extension, which is much larger than the region that is expected to produce the blazar emission at optical/X-ray and gamma-ray energies that we wish to study. However, as reported by Ackermann et al. (2011), there is a correlation between radio and $\mathrm{GeV}$ emission of blazars. In the case of Mrk 501, Ahnen et al. (2017) showed that the radio core emission increased during a period of high gamma-ray activity, 
therefore part of the radio emission seems to be related to the gamma-ray component, and should be considered when studying the blazar emission.

\section{Multiwavelength light curve}

Figure 1 shows a complete set of light curves for all participating instruments, from radio to VHE.

The first panel from the top shows the radio data from the Metsähovi and Owens Valley radio observatories. Each data point represents the average over one night of observations. Optical data in the $R$-band, after host galaxy subtraction as prescribed in Sect. 2.6, are shown in the second panel. The light curve also shows very little variability, with just a slow change in flux of about $\sim 10-20 \%$ on timescales of many tens of days. When compared to the 13 years of optical observations from the Tuorla group ${ }^{9}$, one can note that in 2012 the flux was at a historic minimum. The ultraviolet data from the Swift/UVOT are presented in the third panel and follows the same pattern as the $R$ band fluxes. Overall, the low-frequency observations (radio to ultraviolet) show little variation during this period.

In the X-ray band the Swift/XRT and BAT light curves show a large amount of variation, occurring on timescales of days (i.e. much faster than those in the optical band). The Swift/XRT points represent nightly fluxes derived from $\sim 1 \mathrm{ks}$ observations (where the error bars are smaller than the markers), while the Swift/BAT points are the weighted average of all measurements performed within 5-day intervals. On the day of June 92012 (MJD 56087) a flare is observed where the Swift/XRT flux reached $3.2 \times 10^{-10} \mathrm{erg} \mathrm{cm}^{-2} \mathrm{~s}^{-1}$ in the $2-10 \mathrm{keV}$ band. Interestingly, the largest flux point in the $0.3-2 \mathrm{keV}$ band occurs two days later, indicating that the $\mathrm{X}$-ray activity can have a different variability pattern below and above $2 \mathrm{keV}$.

The Fermi-LAT light curves, which are binned in 7 day time intervals, show some mild variability. The ability to detect small amplitude variability at these energies is strongly limited by the relatively large statistical uncertainty in the flux measurements.

The seventh and eighth panels of Fig. 1 show the VHE light curves from MAGIC, VERITAS and FACT. Here we split the VHE information from MAGIC and VERITAS into two bands, from $200 \mathrm{GeV}$ to $1 \mathrm{TeV}$ and above $1 \mathrm{TeV}$. Each point represents a nightly average, with the 18 MAGIC observations, obtained from an average observation of $1.25 \mathrm{~h}$, and the 28 VERITAS data points, obtained from an average observation of $0.5 \mathrm{~h}$.

The VHE emission is highly variable, with the average in both bands being approximately $0.7 \mathrm{CU}$ above $1 \mathrm{TeV}$. The largest VHE flux is observed on 2012 June 9, where the light curves show a very clear flare (which is also visible in the X-ray light curve) with a $0.2-1 \mathrm{TeV}$ flux of $5.6 \times 10^{-10} \mathrm{~cm}^{-2} \mathrm{~s}^{-1}$ (2.8 CU), and the $>1 \mathrm{TeV}$ flux reaching $1.0 \times 10^{-10} \mathrm{~cm}^{-2} \mathrm{~s}^{-1}$ (4.9 CU). Unfortunately, VERITAS was not scheduled to observe Mrk 501 on June 9.

FACT observed Mrk 501 for an average of $3.3 \mathrm{~h}$ per night over 73 nights during the campaign. As with the other TeV instruments, the data shown are binned nightly.

The FACT fluxes reported in Fig. 1 were obtained with a first-order polynomial that relates the MAGIC flux $\left(\mathrm{ph} \mathrm{cm}^{-2} \mathrm{~s}^{-1}\right.$ above $1 \mathrm{TeV}$ ) and the FACT excess rates (events/hour), as explained in Appendix A.

Measurements of the degree of optical linear polarization and its position angle are displayed in the bottom panels of Fig. 1. As with the optical photometry, the polarization shows

\footnotetext{
9 See http://users.utu.fi/kani/1m/Mkn_501_jy.html
}

only mild variations on time scales of weeks to months during the campaign. Variations of the degree of polarization are muted by the strong contribution of unpolarized starlight from the host galaxy falling within the observation apertures. At these optical flux levels and with the apertures used for the ground-based polarimetry, the optical flux from the host galaxy is about $2 / 3$ of the flux measured, and hence the intrinsic polarization of the blazar is about a factor of three higher than observed. Different instruments used somewhat different apertures and optical bands, which implies that the contribution of the host galaxy to the optical flux and polarization degree will be somewhat different for the different instruments. Since the host galaxy is not subtracted, this leads to small offsets (at the level of $\sim 1 \%$ ) in the measurements of the degree of polarization. The position angle of the polarization (which is not affected by the host galaxy) remains at $120-140^{\circ}$ for more than a month before and after the VHE flare. For comparison, the position angle of the $15 \mathrm{GHz}$ VLBI jet is at $\sim 150^{\circ}$ (Lister et al. 2009). Overall, there is no apparent optical signature, either in flux or linear polarization, that can be associated with the gamma-ray activity observed in Mrk 501 during 2012.

\section{Fractional variability}

In order to characterize the variability at each wavelength we followed the prescription of Vaughan et al. (2003) where the fractional variability $\left(F_{\text {var }}\right)$ is defined as

$$
F_{\mathrm{var}}=\sqrt{\frac{S^{2}-\left\langle\sigma_{\mathrm{err}}^{2}\right\rangle}{\langle x\rangle^{2}}}
$$

Here $S$ is the standard deviation of the flux measurement, $\left\langle\sigma_{\text {err }}^{2}\right\rangle$ the mean squared error and $\langle x\rangle^{2}$ the square of the average photon flux. The error on $F_{\text {var }}$ is estimated following the prescription of Poutanen et al. (2008), as described by Aleksić et al. (2015a)

$\Delta F_{\mathrm{var}}=\sqrt{F_{\mathrm{var}}^{2}+\operatorname{err}\left(\sigma_{\mathrm{NXS}}^{2}\right)}-F_{\mathrm{var}}$

and $\operatorname{err}\left(\sigma_{\mathrm{NXS}}^{2}\right)$ is taken from Eq. (11) in Vaughan et al. (2003)

$\operatorname{err}\left(\sigma_{\mathrm{NXS}}^{2}\right)=\sqrt{\left(\sqrt{\frac{2}{N}} \frac{\left\langle\sigma_{\mathrm{err}}^{2}\right\rangle}{\langle x\rangle^{2}}\right)^{2}+\left(\sqrt{\frac{\left\langle\sigma_{\mathrm{err}}^{2}\right\rangle}{N}} \frac{2 F_{\mathrm{var}}}{\langle x\rangle}\right)^{2}}$

where $N$ is the number of flux measurements. This method, commonly used to quantify the variability, has the caveat that the resulting $F_{\text {var }}$ and its related uncertainty depend on the instrument sensitivity and the observing strategy performed. For instance, densely sampled light curves with small uncertainties in the flux measurements may allow us to see flux variations that are hidden otherwise, and hence may yield a larger $F_{\text {var }}$ and/or smaller uncertainties in the calculated values of $F_{\text {var }}$. This introduces differences in the ability to detect variability in the different energy bands. Issues regarding the application of this method, in the context of multiwavelength campaigns, are discussed by Aleksić et al. (2014, 2015a,b). In the multi-instrument dataset presented in this case, the sensitivity of the instruments Swift/BAT and Fermi-LAT precludes the detection of Mrk 501 on hour timescales, and hence integration over several days is required (and still yields flux measurements with relatively large uncertainties). This means that the Swift/BAT and Fermi-LAT $F_{\text {var }}$ values are not directly comparable to those of the other 


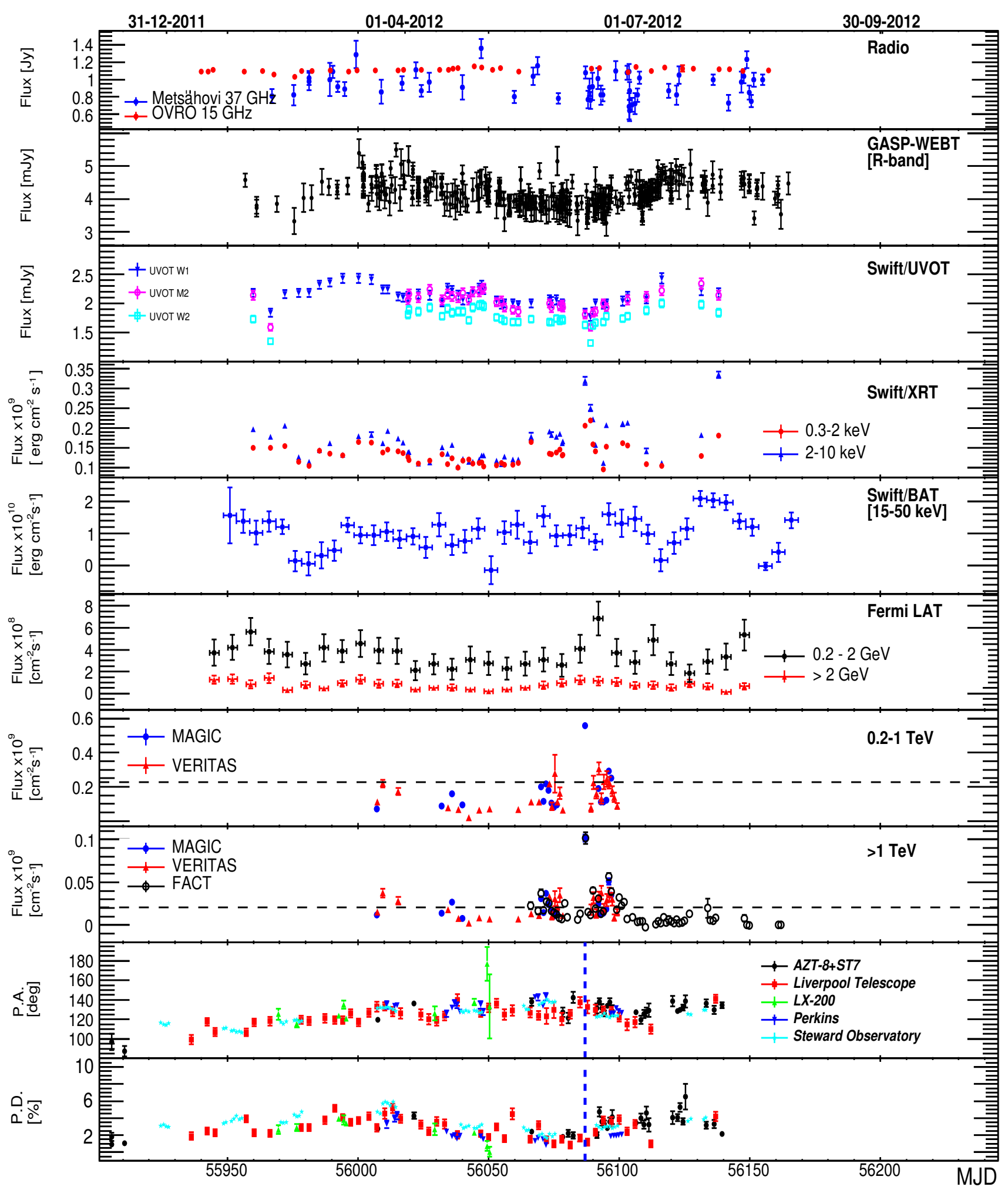

Fig. 1. Multiwavelength light curve for Mrk 501 during the 2012 campaign. The bottom two panels report the electric vector polarization angle (PA) and polarization degree (PD). The correspondence between the instruments and the measured quantities is given in the legends. The horizontal dashed line in the VHE light curves represents $1 \mathrm{CU}$ as reported in Aleksić et al. (2016), and the blue vertical dotted lines in the panels with the polarization light curves depict the day of the large VHE flare (MJD 56087).

instruments, for which $F_{\text {var }}$ values computed with nightly observations (and typically smaller error bars) are reported.

Figure 2 shows the $F_{\text {var }}$ as a function of energy. The left panel uses all the data presented in Fig. 1, with the exception of nights where there were simultaneous FACT and MAGIC data. In these cases the FACT data are removed. The figure displays $F_{\text {var }}$ values for those bands with positive excess variance $\left(S^{2}\right.$ larger than $\left.\left\langle\sigma_{\text {err }}\right\rangle^{2}\right)$; a negative excess variance is interpreted as absence of variability either because there was no variability or because the instruments were not sensitive enough to detect 

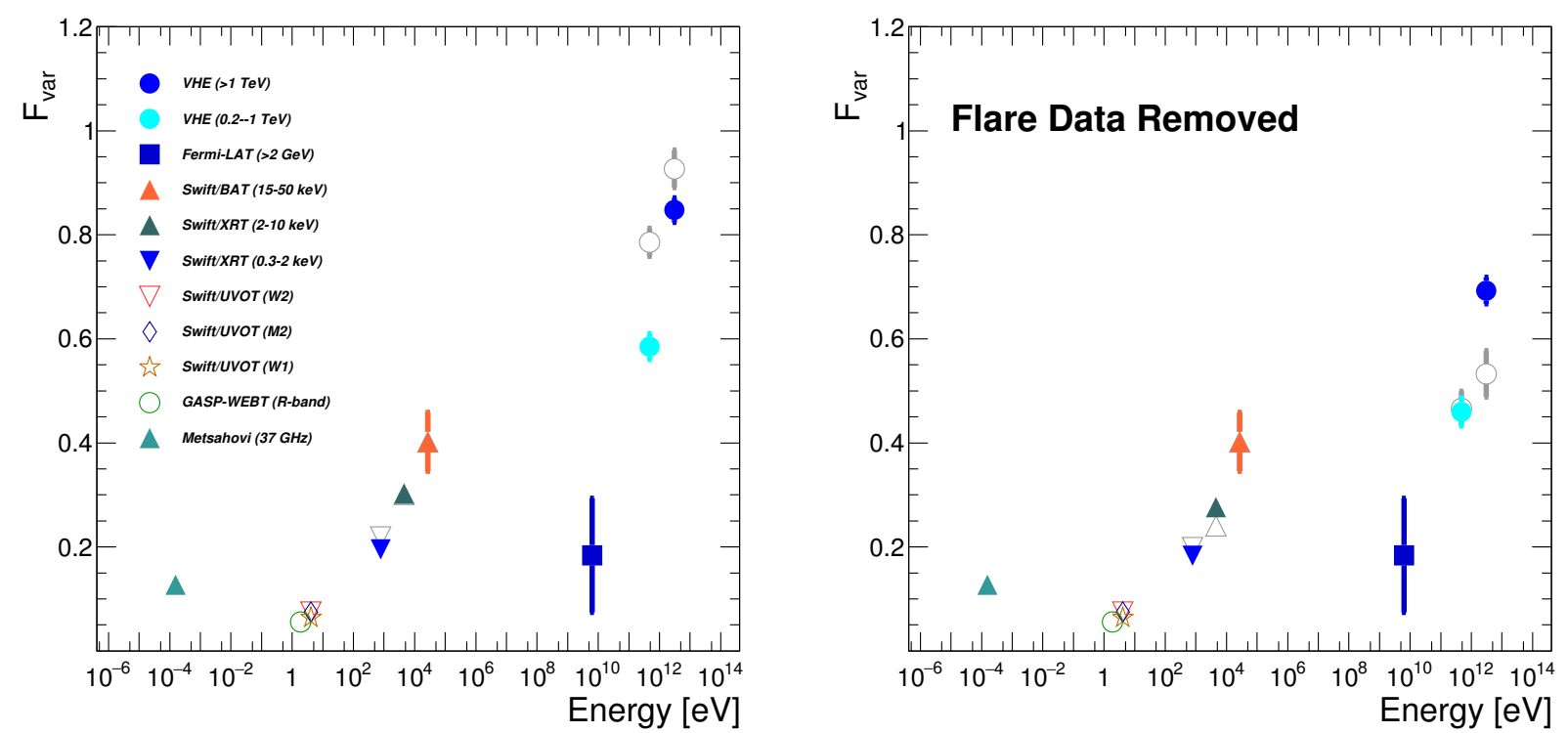

Fig. 2. Fractional variability $F_{\text {var }}$ for each instrument as a function of energy. Left panel includes all data, while the right panel includes all data

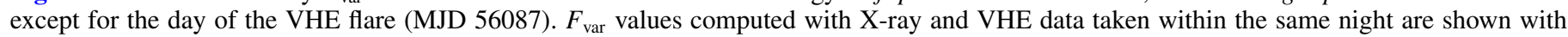
gray open markers.
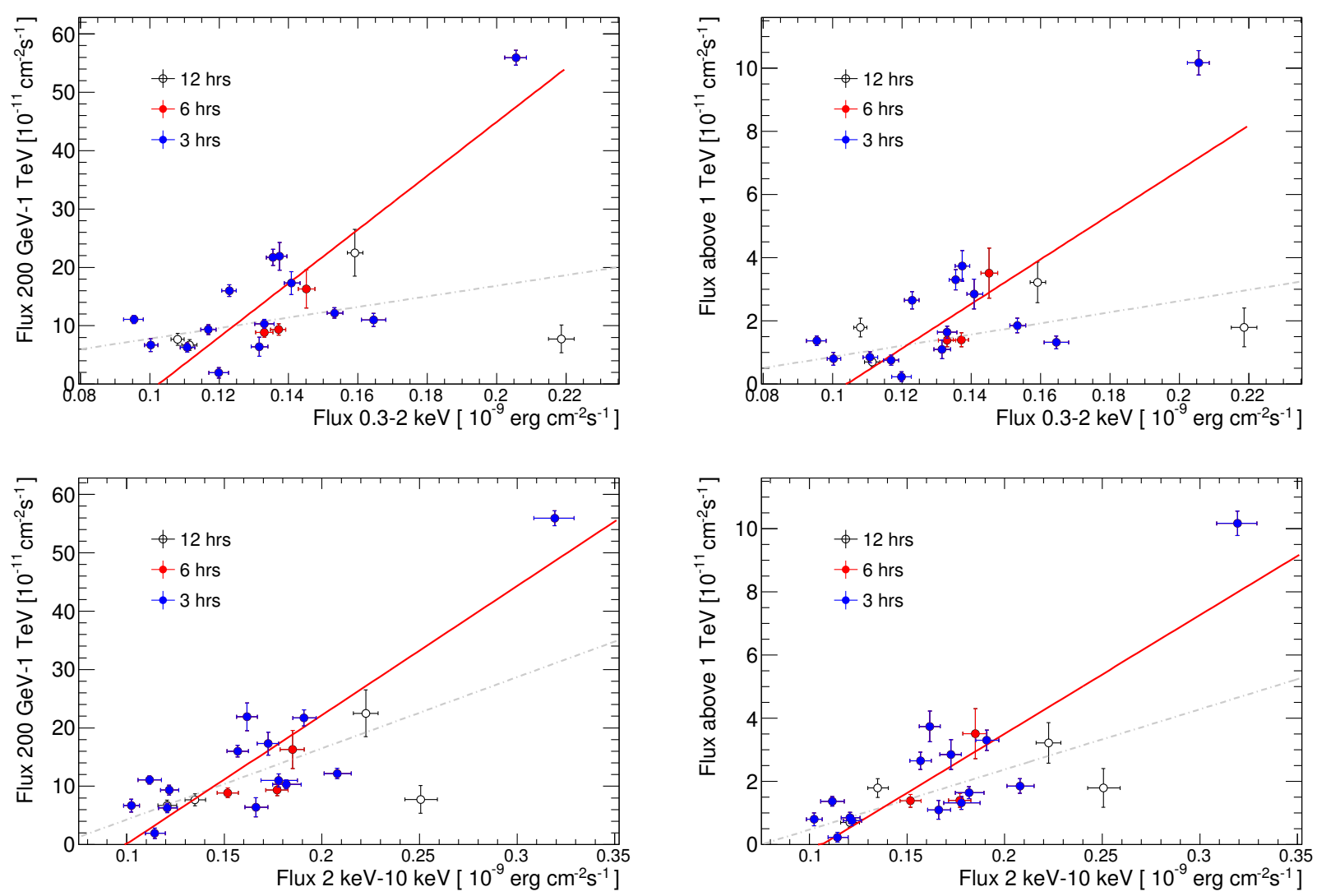

Fig. 3. VHE flux as a function of the Swift/XRT flux for the energy ranges shown. Open circles represent data that were taken within $12 \mathrm{~h}$ of each other, red circles within $6 \mathrm{~h}$ and blue circles within $3 \mathrm{~h}$. In each case, linear fits to the closed circle points (6h or less) are depicted with a red line (when considering the June 9 flare) and with a dotted-dashed gray line (when excluding the June 9 flare).

it. We obtained negative excess variances for the $15 \mathrm{GHz}$ radio fluxes measured with OVRO and the $0.2-2 \mathrm{GeV}$ fluxes measured with Fermi-LAT. The right panel shows the same data except for the flare day (MJD 56087), which has been removed from the multi-instrument dataset, and hence shows a more typical behavior of the source during the 2012 multi-instrument campaign.
Figure 2 also reports the values of $F_{\text {var }}$ obtained by using the X-ray/VHE observations taken simultaneously ${ }^{10}$. Addition-

${ }^{10}$ The $F_{\text {var }}$ in the radio and optical bands does not change much when selecting sub-samples of the full dataset because the variability in these energy bands is small and the flux variations have longer timescales, in comparison with those from the X-ray and VHE bands. 

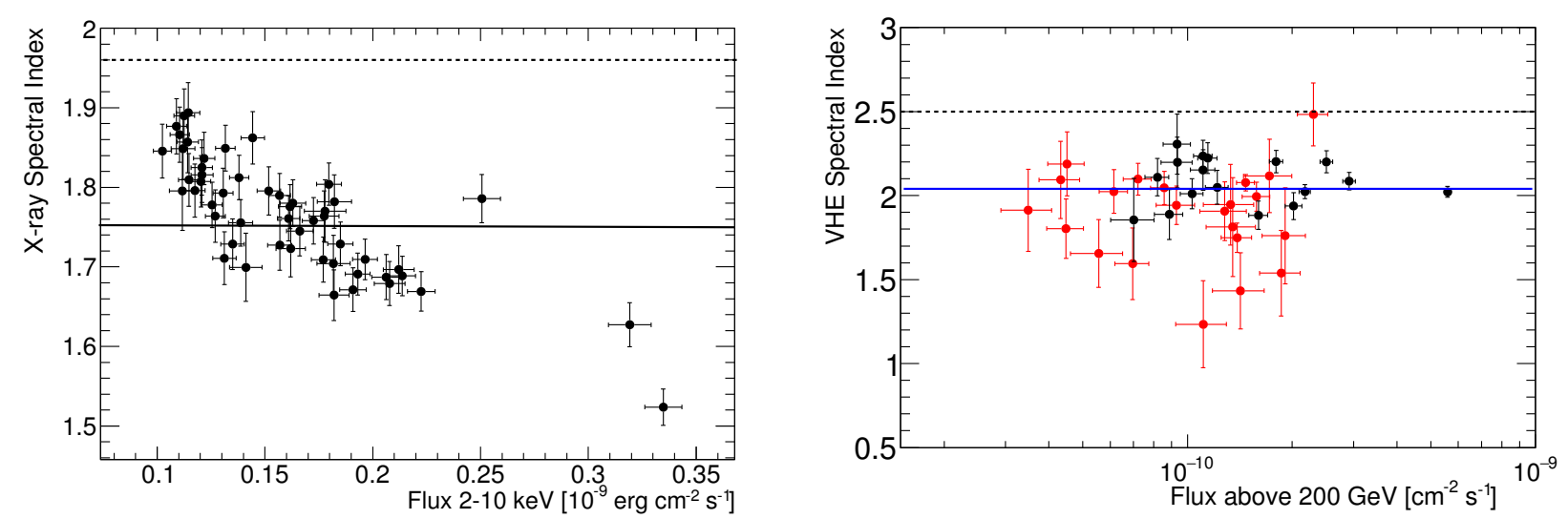

Fig. 4. Left panel: Swift/XRT X-ray power-law spectral index vs flux in the $2-10 \mathrm{keV}$ band. Right panel: measured VHE power-law spectral index vs VHE Flux above $0.2 \mathrm{TeV}$. Red points represent VERITAS data and black points MAGIC data. The data are EBL corrected using Franceschini et al. (2008). The Swift/XRT spectrum of Mrk 501 is often curved, and can be described at keV energies with a spectral index that is typically between 1.8 and 2.1, while the VHE spectral index measured with MAGIC and VERITAS during typical non-flaring activity is about 2.5 (Abdo et al. 2011 ; Aleksić et al. 2015a). The typical spectral indices at X-ray and VHE are marked with a dashed line. For comparison purposes, the panels depict with solid lines the result of a fit with a constant to the X-ray and VHE spectral indices.

Table 1. Correlation results: VHE vs X-ray flux.

\begin{tabular}{ccccccccc}
\hline \hline & \multicolumn{3}{c}{ VHE $(0.2-1 \mathrm{TeV})$} & & \multicolumn{3}{c}{ VHE $(>1 \mathrm{TeV})$} \\
\cline { 2 - 3 } & $\begin{array}{c}\text { Normalized } \\
\text { slope of fit }\end{array}$ & $\begin{array}{c}\text { Pearson correlation } \\
\text { coefficient }(\sigma)\end{array}$ & DCF & & $\begin{array}{c}\text { Normalized } \\
\text { slope of fit }\end{array}$ & $\begin{array}{c}\text { Pearson correlation } \\
\text { coefficient }(\sigma)\end{array}$ & DCF \\
\hline Swift/XRT (0.3-2 keV) & $4.34 \pm 0.19$ & $0.76_{-0.15}^{+0.10}(3.7)$ & $0.72 \pm 0.59$ & & $4.14 \pm 0.27$ & & $0.78_{-0.15}^{+0.10}(3.9)$ & $0.74 \pm 0.59$ \\
Excluding flare & $1.01 \pm 0.21$ & $0.38_{-0.30}^{+0.24}(1.4)$ & $0.37 \pm 0.14$ & & $1.28 \pm 0.25$ & & $0.39_{-0.29}^{+0.32}(1.6)$ & $0.42 \pm 0.17$ \\
Swift/XRT (2-10 keV) & $2.57 \pm 0.13$ & $0.87_{-0.10}^{+0.06}(4.7)$ & $0.81 \pm 0.64$ & & $2.72 \pm 0.16$ & & $0.88_{-0.10}^{+0.06}(4.9)$ & $0.83 \pm 0.64$ \\
Excluding flare & $1.64 \pm 0.20$ & $0.56_{-0.25}^{+0.18}(2.2)$ & $0.54 \pm 0.21$ & & $1.66 \pm 0.21$ & & $0.59_{-0.24}^{+0.16}(2.5)$ & $0.60 \pm 0.20$ \\
\hline
\end{tabular}

Notes. See Sect. 5 and Fig. 3. The normalized slope is the gradient of the fit in Fig. 3, divided by the ratio of the average of each distribution, in order to create a dimensionless scaling factor. Pearson correlation function $1 \sigma$ errors and the significance of the correlation are calculated following Press et al. (2002). Discrete correlation function (DCF) and errors are calculated as prescribed in Edelson \& Krolik (1988).

ally, the right panel in Fig. 2 also shows that, when the large VHE flare from MJD 56087 is removed, the $F_{\text {var }}$ changes substantially in the VHE gamma-ray band (e.g. from $0.93 \pm$ 0.04 down to $0.53 \pm 0.05$ above $1 \mathrm{TeV}$ ) but the variability changes mildly in the X-ray band (e.g. from $0.301 \pm 0.003$ to $0.241 \pm 0.003$ at $2-10 \mathrm{keV})$. In both panels there is a general increase of the fractional variability with increasing energy of the emission. These results will be further discussed in Sect. 7.3.

\section{Correlation between the X-ray and VHE gamma-ray emission}

This section focuses on the cross-correlation between the X-ray and VHE emission, which are the energy bands with the largest variability in the emission of Mrk 501 (as shown in Fig. 2). Figure 3 shows the integral flux for the two VHE ranges, $0.2-1 \mathrm{TeV}$ and $>1 \mathrm{TeV}$, plotted against that for the two Swift/XRT flux bands, $0.3-2 \mathrm{keV}$ and $2-10 \mathrm{keV}$. The symbols are color-coded depending on the time difference between the observations: 3,6 or $12 \mathrm{~h}$. The correlation studies are performed with data taken within $6 \mathrm{~h}$ (the red and blue symbols), which is approximately the largest temporal coverage provided by a Cherenkov telescope for one source during one night.

Three methods were used to test for correlation in each of the four panels shown in Fig. 3, and the results are shown in
Table 1. A Pearson's correlation test was applied to the data and a maximum correlation of $4.9 \sigma$ is found between the higherenergy component of the X-ray band $(2-10 \mathrm{keV})$ and the higherenergy component of the VHE band $(>1 \mathrm{TeV})$. However, this falls to $2.5 \sigma$ when the day of the flare is removed. We also quantified the correlations using the discrete correlation function (DCF, Edelson \& Krolik 1988) which has the advantage over the Pearson correlation that the errors in the individual flux measurements (which contribute to the dispersion in the flux values) are naturally taken into account. Using the data shown in Fig. 3 the correlation for the two higher-energy bands of the X-ray and VHE light curves yields $0.83 \pm 0.64$ when using all data, and $0.60 \pm 0.20$ after removing the June 9 flare. The three-times-larger error in the DCF when the big VHE flare is included is due to the fact that the error in the DCF is given by the dispersion in the individual (for a given pair of X-ray/VHE data points) unbinned discrete correlation function, and this single (flaring) data point deviates substantially from the behavior of the others. The DCF value for the data without the flaring activity corresponds to a marginal correlation at the level of $3 \sigma$, which is consistent with the Pearson correlation analysis. The DCF method is often used to look for a time delay between the emission at different wavelengths. Such a search was carried out for the two X-ray and VHE gamma-ray bands, and no significant delay was found. Neither a linear (shown in Fig. 3) nor a quadratic fit function describes the data well; the linear fit of the highest-energy component in each band, gives a $\chi^{2} /$ d.o.f. of $148.7 / 15$. 
In summary, this correlation study yields only a marginal correlation, which is greatest when comparing the high-energy $\mathrm{X}$-ray and higher-energy VHE gamma-ray components.

In Fig. 4 we present the correlation between the spectral index (derived from a power-law fit) and the integral flux for both the Swift/XRT and VHE data. The spectral fit results with powerlaw functions are reported in Tables B.1, B.2, B.4, respectively for MAGIC, VERITAS and Swift/XRT. At X-rays, the source shows the harder-when-brighter behavior reported several times for Mrk 501 (e.g. Pian et al. 1998; Albert et al. 2007), but such behavior is not observed in the VHE domain during the observing campaign in 2012.

The Mrk 501 spectra measured in the X-ray and VHE ranges were harder than previously observed, during both high and low activity. The very hard X-ray and VHE gamma-ray spectra observed during the full campaign will be further discussed in Sect. 7.1.

\section{Temporal evolution of the broadband spectral energy distribution}

In order to model the data, several time-resolved spectral energy distributions were formed. Spectral measurements were selected in cases where a Swift/XRT spectrum and a MAGIC/VERITAS spectrum were obtained within $6 \mathrm{~h}$ of each other (i.e. from observations performed during the same night). This allowed 17 distinct SEDs to be constructed, spanning three months. The mean absolute time difference between the X-ray and VHE data are $1.2 \mathrm{~h}$, with the maximum time difference being $4.0 \mathrm{~h}$. Because of the substantially lower variability at radio and optical (see Fig. 2) in comparison to that at X-rays and VHE gamma-rays, strict simultaneity in these bands is not relevant. Nevertheless, the $S w i f t / U V O T$ data are naturally simultaneous to that of $S w i f t / X R T$, and the high sampling performed by optical instruments provides a flux measurement well within half day of the X-ray and VHE observations.

\subsection{Theoretical model and fitting methodology}

The broadband SED of Mrk 501 has previously been modeled well using one-zone synchrotron self-Compton (SSC) scenarios during high and low activity (Tavecchio et al. 2001; Abdo et al. 2011; Aleksić et al. 2015a; Furniss et al. 2015). The emission is assumed to come from a spherical region, containing a population of relativistic electrons, traveling along the jet. The region has a radius $R$, is permeated by a magnetic field of strength $B$ and is moving relativistically with a Doppler factor $\delta$.

The electron energy distribution (EED) is assumed to have an energy density $U_{\mathrm{e}}$, and be parameterized by a broken power law with index $p_{1}$ from $\gamma_{1}$ to $\gamma_{b}$ and $p_{2}$ from $\gamma_{b}$ to $\gamma_{2}$, where $\gamma_{i}$ is Lorentz factor of the electrons.

A $\chi^{2}$-minimization fit was performed to find the best-fit SED model to the observed spectra. An SSC code developed by Krawczynski et al. (2004) was incorporated into the XSPEC spectral fitting software (Arnaud et al. 1996) as an external model to perform the minimization using the Levenburg-Marquadt algorithm ${ }^{11}$

In order to decrease the degeneracy among the model parameters, and after inspecting the 17 broadband SEDs, we decided to fix the values of the parameters $\gamma_{\min }, \gamma_{\max }, R$ and $\delta$, and to set the

\footnotetext{
11 https://heasarc.gsfc.nasa.gov/xanadu/xspec/manual/ XSappendixLocal.html
}

location of $\gamma_{\text {brk }}$ to be the cooling break, along with a canonical index change of 1 at $\gamma_{\text {brk }}\left(\right.$ i.e. $\left.p_{2}-p_{1}=1\right)$.

The parameters $\gamma_{\min }, \gamma_{\max }$ are very difficult to constrain with the available broadband SED, as described in Ahnen et al. (2017), and it was decided to fix them to $3 \times 10^{2}$ and $8 \times 10^{6}(\log \gamma=2.5$ and 6.9), which are reasonable values used in the literature (see Abdo et al. 2011; Aleksić et al. 2015a). Additionally, the values of $\delta$ and $R$ were fixed to reasonable values that could successfully describe the data and ensure a minimum variability timescale of 1 day, as no intra-night variability was observed, making this the fastest variability observed during the three-month period considered in this paper. A $\delta \sim 10$ (which results in $R \sim 2.65 \times 10^{16} \mathrm{~cm}$ by variability arguments) is a suitable value used to model the emission of high-peaked BL Lacs such as Mrk 501 (e.g. Ahnen et al. 2017), though it is larger than the modest bulk Lorentz factors suggested by Very Long Baseline Array measurements (Piner et al. 2010; Piner \& Edwards 2004; Edwards \& Piner 2002).

First, we fit the synchrotron peak to adjust the characteristics of the EED and $B$ field. The synchrotron peak is more accurately determined than the inverse-Compton, and has a more direct relation to the EED. Then, we fit the inverse-Compton peak, using all parameters from the fit to the synchrotron peak, and leaving the electron energy density $U_{\mathrm{e}}$ as the only free parameter. After that, we fit the broadband SED using the parameter values from the previous step as starting values. Lastly, we perform a broadband SED fit, using the parameter values from the previous step as starting values, and loosen slightly the condition that the cooling break occurs at $\gamma_{\text {brk }}$, and that the indices in the EED change by exactly 1.0. In this last step, we allow $B$ and $\gamma_{\text {brk }}$ to vary within $\pm 2 \%$, and $p_{1}$ and $p_{2}$ to vary within $\pm 1 \%$ of the values obtained from the previous step. This last step in the fitting procedure provides a non-negligible improvement in the data-model agreement, with minimal (a few \%) departures from the canonical values of $\gamma_{\mathrm{brk}}$ and spectral-index change within the one-zone SSC scenario.

\subsection{Model results}

The results for the 17 broadband SEDs mentioned above can be seen in Figs. 5-7. The corresponding SSC model parameters are listed in Table 2.

We found that the one-zone SSC model approximately describes the X-ray and VHE gamma-ray data. However, the model is not able to produce sufficient emission at $\mathrm{eV}$ energies to describe the optical-UV emission and the soft X-ray emission with a single component. A similar problem in modeling the broadband SED of Mrk 501 within a one-zone SSC framework was reported in Ahnen et al. (2017). During 2012, the variability in the optical-UV band was less than 10\%, as reported in Sect. 4, and the $R$-band flux was at a historical minimum (over 13 years of observations performed by the Tuorla group), as mentioned in Sect. 3. It is therefore reasonable to assume that this part of the spectrum is dominated by the emission from a distinct region of the jet, where the emission is slowly changing on timescales of many weeks. This new region, if populated by high electron density, could also contribute to the $\mathrm{GeV}$ emission. But this contribution should be characterized by low flux variability (lower than the one measured), as occurs in the optical emission. For simplicity, we will not consider the description of the optical-UV emission in the theoretical scenario presented here, which focuses on the Xray and VHE gamma-ray bands, that is, the most variable portions of the electromagnetic spectrum and where most of the energy is emitted.

While only the X-ray and VHE data are strictly simultaneous (within four hours) and therefore used in the one-zone SSC model 

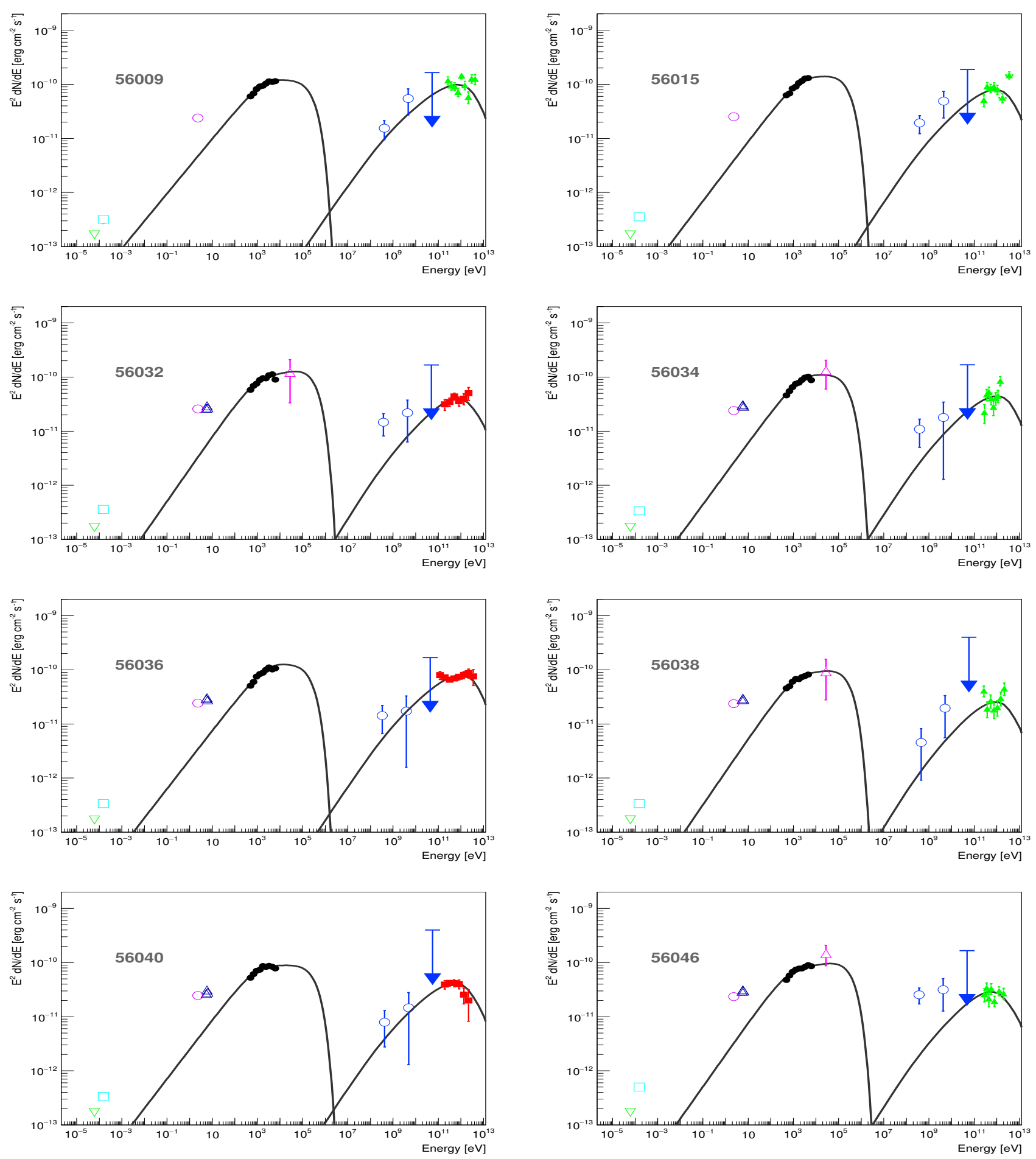

Fig. 5. Spectral energy distributions (SED) for 8 observations between MJD 56009 and MJD 57046. The markers match the following experiments; the green open triangle OVRO (radio $15 \mathrm{GHz}$ ), blue open square Metshovi (radio $37 \mathrm{GHz}$ ), red open circle ( $R$-band optical, corrected for host galaxy), blue open triangles Swift/UVOT (UV), black filled circles Swift/XRT (X-ray), pink open triangles Swift/BAT (X-ray), blue open circles Fermi-LAT (gamma rays) and red/green filled squares/triangles MAGIC/VERITAS (VHE gamma rays). VHE data are EBL-corrected using Franceschini et al. (2008). The BAT energy flux relates to a one-day average, while the Fermi-LAT energy flux relates to three-day average centered at the VHE observation. Filled markers are those fit by the theoretical model, while open markers are not. The black line represents the best fit with a one-zone SSC model, with the results of the fit reported in Table 2.

fits, we note that the three-day average $\mathrm{GeV}$ emission (centered on the VHE observation) measured with Fermi-LAT matches well most model curves on a case-by-case basis. The notable exceptions are on MJD 56046 and MJD 56095, where the LAT spectral points (especially the one at the lower energy) deviate from the theoretical curve, worsening the $\chi^{2} /$ d.o.f. of the fit from $23.5 / 12$ to $33.0 / 14$ for the first day and from $16.8 / 10$ to $27.2 / 12$ for the second one. The combination of the LAT and MAGIC/VERITAS spectral points for these two days shows a flat gamma-ray bump over four orders of magnitude (from $0.2 \mathrm{GeV}$ to $2 \mathrm{TeV}$ ). The $p$ values of those fits, when considering also the agreement with the two LAT data points, are $0.3 \%$ and $0.7 \%$, which is compa- 

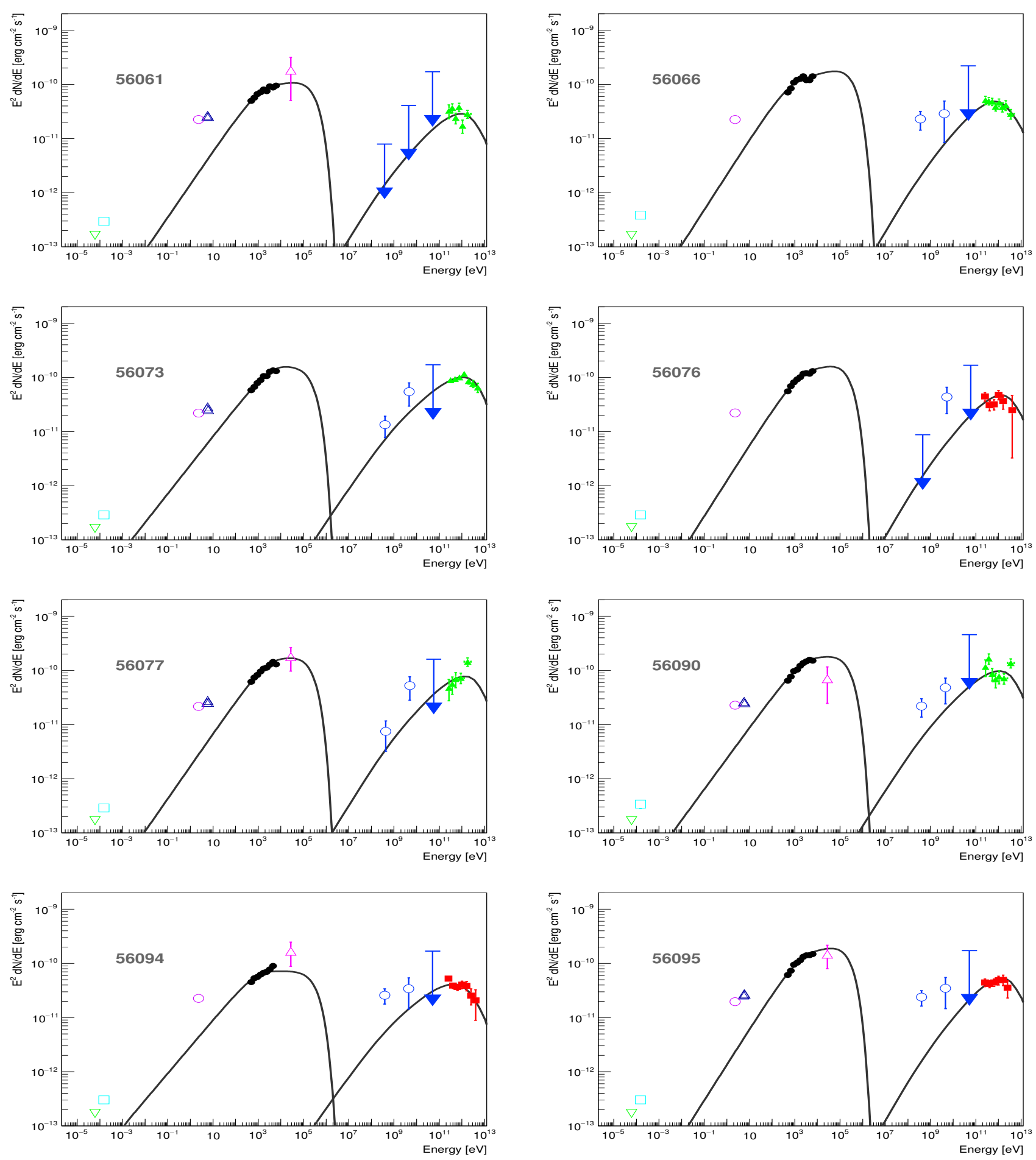

Fig. 6. Spectral energy distributions (SED) for 8 observations between MJD 57061 and MJD 57095. See Fig. 5 for explanation of markers and other details. The black line represents the best fit with a one-zone SSC model, with the results of the fit reported in Table 2.

rable to the data-model agreement from other broadband SEDs where the LAT spectral points match well with the model curves (e.g. MJD 56015, 56034). These two broadband SEDs may hint at the existence of an additional component emitting at $\mathrm{GeV}$ energies, as has already been proposed by Shukla et al. (2015). However, using the data presented in this paper, the statistical significance is not large enough to make that claim, and we will not consider additional (and variable) GeV components in our theoretical model. On the other hand, it is also worth noticing that most of the Fermi-LAT data points are systematically located above (within 1-2 $\sigma$ ) the SSC model curves, which may be taken as another hint for the existence of an additional contribution at $\mathrm{GeV}$ energies that is constantly present at some level.

From the fit parameters, we can derive a value for $\eta$, the ratio of the electron energy density to the magnetic field energy density, which gives an indication of the departure from equipartition (Tavecchio \& Ghisellini 2016). Here the values differ from unity by more than two orders of magnitude, indicating that the particle population has an excess of energy compared to the magnetic 

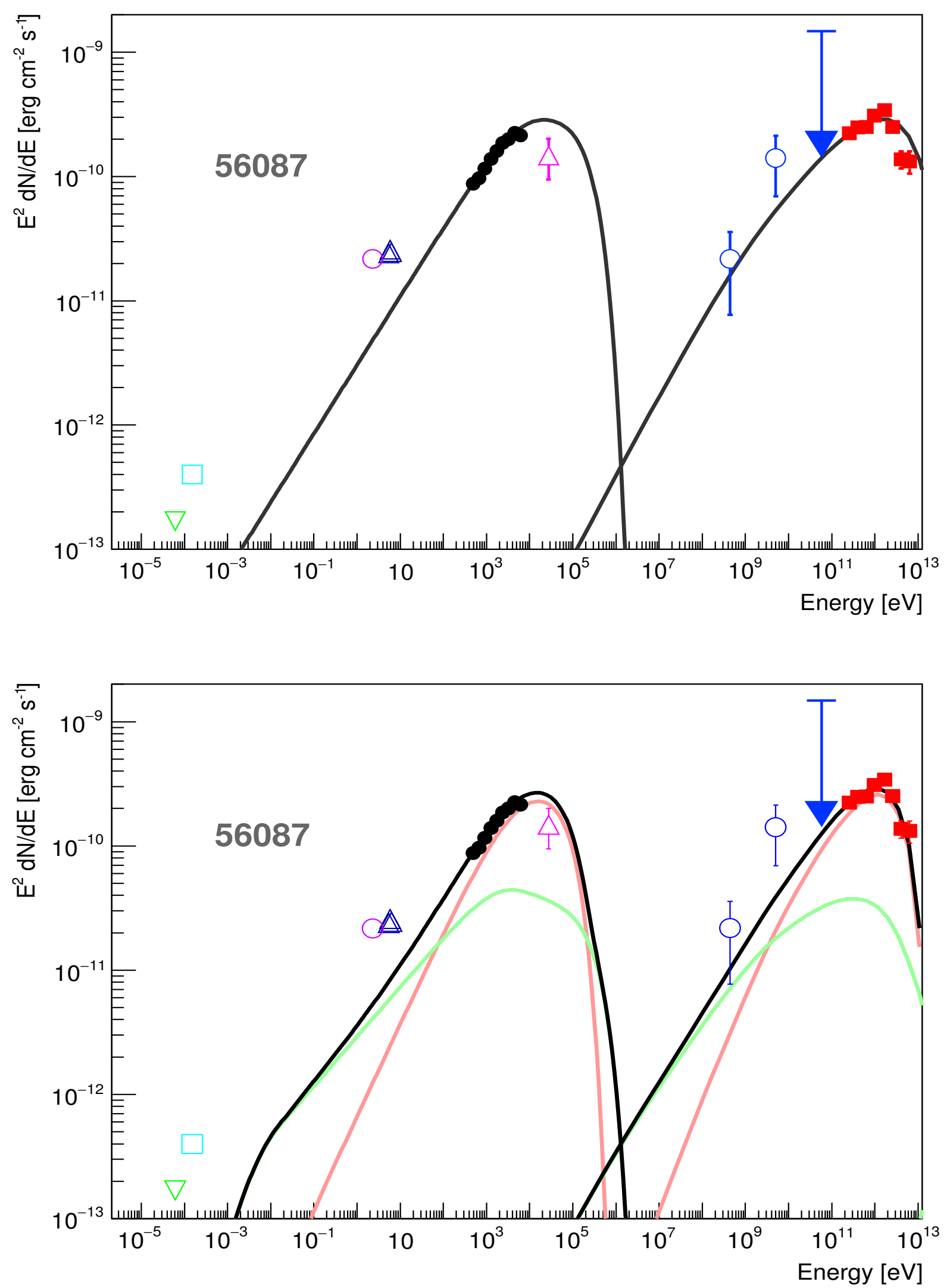

Fig. 7. Broadband SED for MJD 56087 (VHE flare from 2012 June 9), fitted with a one-zone SSC model (top panel) and a two-zone SSC model (bottom panel). See Fig. 5 for explanation of markers and other details. In the bottom panel, the green line depicts the emission of the first (large) zone responsible for the baseline emission, and the red line the emission from the second (smaller) zone, that is responsible for the flaring state. The fit results from the one-zone SSC model fit are reported in Table 2, while those from the two-zone SSC model fit are reported in Table 3. 
Table 2. One-zone SSC model results.

\begin{tabular}{|c|c|c|c|c|c|c|}
\hline $\operatorname{MJD}\left(\chi^{2} /\right.$ d.o.f. $)$ & $\begin{array}{c}B \\
\left(10^{-2} \mathrm{G}\right)\end{array}$ & $\begin{array}{c}\gamma_{\text {brk }} \\
\left(10^{6}\right) \\
\end{array}$ & $p_{1}$ & $p_{2}$ & $\begin{array}{c}U_{\mathrm{e}} \\
\left(10^{-3} \mathrm{erg} \mathrm{cm}^{-3}\right)\end{array}$ & $\begin{array}{c}\eta \\
{\left[U_{\mathrm{e}} / U_{\mathrm{B}}\right]}\end{array}$ \\
\hline $56009 \mathrm{~V}(34.0 / 13)$ & 2.26 & 0.85 & 1.90 & 2.87 & 11.96 & 589 \\
\hline 56015 V (29.9/11) & 2.34 & 0.81 & 1.90 & 2.87 & 9.27 & 425 \\
\hline $56032 \mathrm{M}(19.9 / 10)$ & 2.99 & 0.49 & 1.88 & 2.77 & 5.20 & 146 \\
\hline $56034 \mathrm{~V}(24.3 / 12)$ & 2.22 & 0.90 & 1.86 & 2.90 & 6.88 & 350 \\
\hline $56036 \mathrm{M}(21.0 / 11)$ & 2.00 & 1.07 & 1.93 & 2.96 & 10.50 & 659 \\
\hline 56038 V (19.8/10) & 2.55 & 0.63 & 1.78 & 2.82 & 4.50 & 173 \\
\hline $56040 \mathrm{M}(18.8 / 11)$ & 3.00 & 0.51 & 1.91 & 2.93 & 5.98 & 166 \\
\hline $56046 \mathrm{~V}(23.5 / 12)$ & 3.26 & 0.41 & 1.81 & 2.82 & 4.30 & 102 \\
\hline $56061 \mathrm{~V}(24.0 / 10)$ & 2.65 & 0.65 & 1.78 & 2.82 & 4.66 & 166 \\
\hline $56066 \mathrm{~V}(36.0 / 12)$ & 3.39 & 0.42 & 1.70 & 2.73 & 5.11 & 112 \\
\hline $56073 \mathrm{~V}(13.3 / 11)$ & 2.00 & 1.28 & 1.93 & 2.96 & 11.70 & 736 \\
\hline $56076 \mathrm{M}(19.7 / 10)$ & 2.13 & 0.81 & 1.69 & 2.70 & 6.57 & 361 \\
\hline 56077 V (17.7/9) & 1.96 & 1.07 & 1.80 & 2.82 & 9.29 & 607 \\
\hline $56087 \mathrm{M}(62.5 / 12)$ & 1.64 & 1.70 & 1.89 & 2.91 & 21.30 & 1398 \\
\hline $56090 \mathrm{~V}(32.7 / 10)$ & 2.21 & 0.91 & 1.86 & 2.83 & 10.10 & 520 \\
\hline $56094 \mathrm{M}(18.0 / 10)$ & 2.98 & 0.50 & 2.00 & 2.97 & 7.04 & 199 \\
\hline $56095 \mathrm{M}(16.8 / 10)$ & 2.25 & 0.84 & 1.68 & 2.73 & 6.78 & 336 \\
\hline
\end{tabular}

Notes. The following parameters were fixed: region size $(R) 2.65 \times 10^{16} \mathrm{~cm}$, the Doppler factor $(\delta) 10, \gamma_{\min } 3.17 \times 10^{2}$ and $\gamma_{\max } 7.96 \times 10^{6}$. V refers to VERITAS and M to MAGIC observations.

Table 3. Two-zone SED model results.

\begin{tabular}{lcccccc}
\hline \hline MJD ( $\chi^{2} /$ d.o.f. $)$ & $\begin{array}{c}B \\
\left(10^{-2} \mathrm{G}\right)\end{array}$ & $\begin{array}{c}\gamma_{\mathrm{brk}} \\
\left(10^{6}\right)\end{array}$ & $p_{1}$ & $p_{2}$ & $\begin{array}{c}U_{\mathrm{e}} \\
\left(10^{-3} \mathrm{erg} \mathrm{cm}^{-3}\right)\end{array}$ & $\begin{array}{c}\eta \\
\left(U_{\mathrm{e}} / U_{\mathrm{B}}\right)\end{array}$ \\
\hline Quiescent state & 2.1 & 1.0 & 2.17 & 3.18 & 14.1 & 775 \\
56087 M (31.2/7) & 6.8 & 0.74 & 1.50 & 2.52 & 420 & 2280 \\
\hline
\end{tabular}

Notes. The fixed parameters are the same as in Table 2 except for the size and the energy span of the EED for the flaring zone, which are $R=$ $3.3 \times 10^{15} \mathrm{~cm}, \gamma_{\min }=2 \times 10^{3}$ and $\gamma_{\max }=2 \times 10^{6}$.

field. This is a common situation when modeling the broadband SEDs of Mrk 501 (and TeV blazars in general) with a onezone SSC scenario (see Tavecchio et al. 2001; Abdo et al. 2011; Aleksić et al. 2015a; Furniss et al. 2015), which implies more energy in the particles than in the magnetic field, at least locally where the broadband blazar emission is produced. It is interesting to note that Baring et al. (2017) employ complete thermal plus non-thermal distributions in their shock acceleration modeling of Mrk 501 (2009 campaign) and other blazar multiwavelength spectra, determining $U_{\mathrm{e}}$ consistently, and, using a $B$ field of $\sim 10^{-2} \mathrm{G}$, arrive at a value of $\eta \sim 300$ for Mrk 501, which is very similar (within a factor of $\sim 2$ ) to the values reported in Table 2 of this manuscript.

The worst SSC model fit by far is the one for MJD 56087 (2012 June 9), where $\chi^{2} /$ d.o.f. $=62.5 / 12\left(p=8 \times 10^{-9}\right)$. This day corresponds to the large VHE gamma-ray flare reported in Sect. 3, for which the SED shows a peak-like structure centered at $\sim 2 \mathrm{TeV}$.

For the sake of completeness, we attempted a fit leaving all the model parameters free, apart from the relation between $R$ and Doppler factor to ensure a minimum variability of 1 day. This fit yielded a $\chi^{2} /$ d.o.f. $=30.3 / 9\left(p=4 \times 10^{-4}\right)$. While this fit provides a better data-model agreement, the obtained model is less physically meaningful because the model parameters are not related as expected in the canonical one-zone SSC framework (e.g. $\gamma_{\mathrm{br}}$ and $B$, or $p_{1}$ and $p_{2}$ ). Moreover, this fit requires a $\gamma_{\min }=6 \times 10^{4}$, which is an unusually high value for HBLs such as Mrk 501. Because of that, we attempted a fit with a two-zone SSC sce- nario with model parameters physically related as we did for the one-zone SSC scenario described in Sect. 6.1. In this framework, one relatively large zone dominates the emission at optical and $\mathrm{MeV}$ energies (and is presumed steady or slowly changing with time). The other, smaller zone, which is spatially separated from the first, is characterized by a very narrow electron energy distribution and dominates the variable emission occurring at X-rays and VHE gamma rays, and eventually also produces narrow inverse-Compton bumps. This scenario was successfully used to model a 13-day-long period of flaring activity in Mrk 421, as reported in Aleksić et al. (2015c). To describe the broadband SED of Mrk501 measured for MJD 56087, the EED of the second region was chosen to span over three orders of magnitude, from $\gamma_{\min }=2 \times 10^{3}$ to $\gamma_{\max }=2 \times 10^{6}$, and to have a radius $R$ of $3.3 \times 10^{15} \mathrm{~cm}$ which, for a Doppler factor of 10 , corresponds to a light-crossing time of three hours, and hence suitable to describe variability with timescales much shorter than one day. The broadband SED fitting using the two-zone SSC model is done in the same way as the one-zone SSC model fit described above, but now with twice as many parameters. The resulting model fit is displayed in Fig. 7, and the model parameters reported in Table 3 . The data-model agreement achieved with this two-zone SSC scenario yielded $\chi^{2} /$ d.o.f. $=31.2 / 7(p=6 \times$ $10^{-5}$ ) which, although this scenario still does not describe the broadband data satisfactorily, is still several orders of magnitude better than the $\mathrm{p}$ value obtained with a single-zone SSC scenario. 


\section{Discussion}

\subsection{Mrk 501 as an Extreme BL Lac object in 2012}

The BL Lac objects known to emit VHE gamma rays have the maximum of their high-energy component typically peaking in the $1-100 \mathrm{GeV}$ band, which implies that IACTs measure soft VHE spectra (power-law indices $\Gamma>2$, where $\left.\mathrm{d} N / \mathrm{d} E \propto E^{-\Gamma}\right)$. However, there is also a small number of VHE BL Lacs where the maximum of the gamma-ray peak is located well within the VHE band (Tavecchio et al. 2011), which implies that IACTs would measure hard VHE spectra (power-law indices $\Gamma<2$ ), once the spectra are corrected for the absorption in the EBL. These objects have the peak of their synchrotron peak also at higher energies $(>1-10 \mathrm{keV})$, a property which was initially used to flag them as special sources, and categorize them as "extreme HBLs" (EHBLs, Costamante et al. 2001). Archetypal objects belonging to this class, and extensively studied in the last few years, are 1ES 0229+200 (Aharonian et al. 2007b; Vovk et al. 2012; Aliu et al. 2014; Cerruti 2013) and 1ES 0347-121 (Aharonian et al. 2007a; Tanaka et al. 2014). Some of the sources classified as EHBLs according to the position of their synchrotron peak have been shown to have a very soft VHE gamma-ray spectrum (e.g. RBS 0723, Fallah Ramazani 2017), which indicates that there is not a uniform class of EHBLs, and hence some diversity within this classification of sources (entirely based on observations). In this section we focus on those EHBLs that also have a hard VHE gamma-ray component (e.g. 1ES 0229+200), which are actually the most relevant objects for EBL and intergalactic magnetic field (IGMF) studies (Domínguez \& Ajello 2015; Finke et al. 2015)

In order to model the broadband SEDs of these EHBLs with hard VHE gamma-ray spectral components, one requires special physical conditions (see e.g. Tavecchio et al. 2009; Lefa et al. 2011; Tanaka et al. 2014), such as large minimum electron energies $\left(\gamma_{\min }>10^{2-3}\right)$ and low magnetic fields $(B \lesssim 10-20 \mathrm{mG})$. Moreover, leptonic models are also challenged by the limited variability of the VHE emission of EHBLs, which differs very much from the typically high variability observed in the VHE emission of HBLs. For that reason, several authors have proposed that the VHE gamma-ray emission is the result of electromagnetic cascades occurring in the intergalactic space, possibly triggered by a beam of high-energy hadrons produced in the jet of the EHBLs (e.g. Essey \& Kusenko 2010), or alternatively produced within leptohadronic models (e.g. Cerruti et al. 2015).

Aside from its importance in blazar emission models, the extremely hard gamma-ray emission allows constraints to be placed on the IGMF (e.g. Neronov \& Vovk 2010), and provides a powerful tool to study the absorption of gamma rays in the EBL (e.g. Costamante 2013). Potential deviations from this absorption are also of interest, as they could be related to the mixing of photons with new spin-zero bosons such as axion-like particles (e.g. de Angelis et al. 2007, 2011; SánchezConde et al. 2009).

Therefore, it is evident that EHBLs with hard VHE gammaray spectral components are fascinating objects that can be used to study blazar jet phenomenology, high-energy cosmic rays, EBL and IGMF. The main problem is that there are only a few sources identified as EHBLs and detected using IACTs and that they are typically rather faint. implying the need for very long observations, which complicates the studies mentioned above. For instance, 1ES $0229+200$, which is probably the most studied EHBL, has a VHE flux above $580 \mathrm{GeV}$ of only $\sim 0.02 \mathrm{CU}$, and for many years it was thought to be a steady gamma-ray source. A $130 \mathrm{~h}$ observation performed by H.E.S.S. recently showed that the source is variable (Cologna et al. 2015), which has strong implications for example on the lower limits derived on the IGMF.

Mrk 501 has been observed for a number of years by MAGIC and VERITAS, and it has typically shown a soft VHE gammaray spectrum, with a power-law index $\Gamma \sim 2.5$ (e.g. Abdo et al. 2011; Acciari et al. 2011a; Aleksić et al. 2015a). It is known that during strong gamma-ray activity, such as the activity in 1997 and 2005, the VHE spectra became harder, with $\Gamma \sim 2.1-$ 2.2 (DjannatiAtai et al. 1999; Samuelson et al. 1998; Albert et al. 2007) and recently Aliu et al. (2016) have also reported similar spectral hardening during the outstanding activity in May 2009. It is worth noticing that during the big flare in April 1997, the synchrotron peak of Mrk501 shifted to energies beyond $100 \mathrm{keV}$, and that Mrk 501 was identified as an EHBL by Costamante et al. (2001). However, this happened only during extreme flaring events. On the contrary, as displayed in Fig. 4, during this campaign, Mrk 501 shows very hard X-ray and VHE gamma-ray spectra during both very high and the quiescent or low activity. The measured VHE spectra show power-law indices harder than 2.0, which has never been measured before, and the hardness of the VHE spectrum is independent of the measured activity. A fit to the spectral indices with a constant yields $\Gamma=2.041 \pm 0.015\left(\chi^{2} / \mathrm{NDF}\right.$ $=86 / 38)$. The left panel of Fig. 4 shows an average X-ray spectral index value of $1.752 \pm 0.004\left(\chi^{2} / \mathrm{NDF}=330 / 51\right)$. In both cases we have clear spectral variability, hence spectra which statistically differ from the mean value. In contrast to the VHE spectra, in the $\mathrm{X}$-ray spectra one can observe a dependence on the source activity, with the spectrum getting harder with increasing flux; but Mrk 501 shows spectra with photon index $<2.0$ even for the lowest-activity days. We did not find any relation between the X-ray and VHE spectral indices.

In summary, during the 2012 campaign, both the X-ray and VHE spectra were persistently harder than $\Gamma=2$ (during low and high source activity), which implies that the maximum of the synchrotron peak is above $5 \mathrm{keV}$, and the maximum of the inverseCompton peak is above $0.5 \mathrm{TeV}$. In other words, Mrk 501 behaved effectively like an EHBL during 2012. This suggests that being an EHBL may not be a permanent characteristic of a blazar, but rather a state which may change over time.

\subsection{Model for the temporal evolution of the broadband SED}

The accurate description of the broadband SED of Mrk 501 and its temporal evolution can be provided by a complex theoretical scenario involving the superposition of several emitting regions, as reported in Sect. 6. We have shown that the opticalUV emission and the soft X-ray emission cannot be parameterized with a single synchrotron component, something that had already been observed during the campaign from 2009 (Ahnen et al. 2017). Additionally, the 3-day-integrated GeV emission, as measured by Fermi-LAT, is systematically above (at 1-2 $\sigma$ for single SEDs) the model curves and, in two SEDs, we found indications of an additional component at $\mathrm{MeV}-\mathrm{GeV}$ energies, something which had been also reported by Shukla et al. (2015) using observations from 2010 and 2011. However, the X-ray and VHE gamma-ray bands, which are the segments of the SED with the highest energy flux, and the most variable ones, can be described in a satisfactory way with a simple one-zone SSC model. This fact allows one to draw straightforward physical conclusions with a reduced number of model parameters.

The electron spectral indices vary slightly across the models while the break energy changes by a factor of three, reaching the highest value during the night of the flare. The particle spectra 
were found to be hard, with $p_{1} \leq 2$ for most cases, which is needed to explain the very hard X-ray and VHE spectra. We also find a strong positive correlation between the electron energy density, $U_{\mathrm{e}}$ (derived from the one-zone SSC model) and the VHE gammaray emission measured by MAGIC and VERITAS. The Pearson's correlation coefficient between $U_{\mathrm{e}}$ and both the $0.2-1 \mathrm{TeV}$ and above $1 \mathrm{TeV}$ flux is $0.97_{-0.02}^{+0.01}$, with the significance of the correlation being larger than $7 \sigma$. The value of $U_{\mathrm{e}}$ depends on the value used for $\gamma_{\min }$, which is not well constrained by the data. But we noted that $U_{\mathrm{e}}$ only changes by $10-20 \%$ when changing $\gamma_{\min }$ by one order of magnitude. Given that the SSC modeling requires changes in $U_{\mathrm{e}}$ by factors of a few to explain the 17 broadband SEDs of Mrk 501, we consider that the dependency on the chosen value for $\gamma_{\min }$ does not have any relevant impact in the significant correlation between the measured VHE flux and the SSC model $U_{\mathrm{e}}$ values. This relation indicates that, within the one-zone SSC used here, the main cause of the broadband SED variability is the injection or acceleration of electrons.

The average broadband SED of Mrk 501 during the observing campaign in 2009 was successfully modeled with a one-zone SSC scenario, where the energisation of the electrons was attributed to diffusive first-order Fermi acceleration (Abdo et al. 2011). Yet during the multi-instrument observations in 2012 we measured substantially harder X-ray and VHE spectra that required EEDs with harder spectra in the models. Such hard-spectrum EEDs may be produced through second-order Fermi acceleration (Chen et al. 2015; Lefa et al. 2011; Tammi \& Duffy 2009; Shukla et al. 2016).

Additionally, the radiative cooling of a monoenergetic pileup particle energy distribution can result in a power-law particle distribution with index of 2 (Saugé \& Henri 2004). These narrow distributions of particles may arise through stochastic acceleration by energy exchanges with resonant Alfvén waves in a turbulent medium as described by Schlickeiser (1985), Stawarz \& Petrosian (2008), and Asano et al. (2014). In this case quasi-Maxwellian distributions are obtained: these have been suggested by multiwavelength modeling of Mrk 421 (Aleksić et al. 2015c). Magnetic reconnection in blazar jets (Giannios et al. 2010), which has been invoked by Paliya et al. (2015) to explain the variability of Mrk 421, is another process that can effectively produce hard EEDs (Cerutti et al. 2012a,b). As reported in Zhang et al. (2014, 2015), through magnetic reconnection, the dissipated magnetic energy is converted into non-thermal particle energy, hence leading to a decrease in the magnetic field strength $B$ for increasing gamma-ray activity and $U_{\mathrm{e}}$. This trend is also observed in the parameter values retrieved from our SSC model parameterisation (see Table 2), thus supporting the hypothesis of magnetic reconnection occurring in the jets of Mrk 501.

In principle, obtaining hard EEDs from a diffusive shock acceleration process is difficult, as first-order Fermi acceleration produces a power-law index with value of 2 , and the spectrum then evolves in time due to radiative cooling and steepens further. However, Baring and collaborators (Baring et al. 2017) have recently shown that shock acceleration can also produce hard EEDs with indices as hard as one, primarily because of efficient drift acceleration in low levels of MHD turbulence near relativistic shocks: see Summerlin \& Baring (2012) for a complete discussion.

As reported in Sect. 6, the X-ray and gamma-ray segments from the SED related to the large VHE flare on MJD 56087 (2012 June 9) were modeled with a two-zone SSC model in order to better describe the high-energy peak, with a maximum at $\sim 2 \mathrm{TeV}$. In this scenario, the X-ray and VHE spectra are completely dominated by the emission of a region that is smaller (by one order of magnitude), and with a narrower EED characterized by a very high minimum Lorentz factor $\gamma_{\min }$. This multizone SSC scenario was successfully used to model the broadband SEDs of Mrk 421 that also showed peaked or multipeaked structures during a 13-day period of flaring activity in March 2010 (Aleksić et al. 2015c). The relatively steady optical and $\mathrm{GeV}$ emission could be produced in a shock-in-jet component while the variable $\mathrm{X}$ ray and VHE gamma-ray emission could arise from a component originating in the base of the jet and producing this relatively narrow EED. The more compact zone is probably intimately connected to the injector site, perhaps a jet shock, thereby more directly sampling the acceleration characteristics since there has been less time for electrons to cool in the ambient magnetic field (Baring et al. 2017).

It is also worth noting that the large VHE flare from June 9 2012 occurred when the degree of polarization was at its lowest value ( $2 \%$ ) during the 2012 campaign (see bottom panels of Fig. 1). On the other hand, the large VHE flare from May 12009 occurred when the degree of polarization was at its highest value ( 5\%) during the 2009 campaign (Aliu et al. 2016; Ahnen et al. 2017). Since enhanced polarization is naturally anticipated in short duration flares where smaller length scales are sampled, this observational dichotomy complicates the picture. This observation suggests that there is a diversity in gamma-ray flares in Mrk 501, and at least some of them seem not to involve any change in the degree of polarization, which may occur naturally if the optical and the VHE emission are produced in different regions of the jet.

\subsection{Multiband variability and correlations}

Section 4 reports a general increase in the flux variability with increasing energy. At radio, optical and UV bands we observe relatively low variability $\left(F_{\mathrm{var}} \leq 0.1\right)$, but for the variability at the $37 \mathrm{GHz}$ radio fluxes from Metsahovi, which is $0.13 \pm 0.02$. This variability is not produced by a flare, or by a slow temporal evolution (weeks or months long) of the light curve, which is often observed at radio, but by a consistent flickering in the radio fluxes. Such flickering is rare in blazars, but it has been already reported in previous observing campaigns of Mrk 501 (Aleksić et al. 2015a; Furniss et al. 2015). In the $\mathrm{X}$-rays and $\mathrm{GeV}$ gamma-ray bands we observe high variability $\left(F_{\text {var }} \sim 0.2-0.4\right)$. However, we note that we do not have sensitivity to determine the fractional variability in the band $0.2-2 \mathrm{GeV}$ (where the excess variance is negative), and hence the fractional variability in this band could be lower than that measured at X-rays. In the VHE gamma-ray band we observe very high variability $\left(F_{\text {var }} \sim 0.5-0.9\right)$, i.e. about three times larger than that at X-rays.

Such a multiband (from radio to VHE) variability pattern was first reported with observations from 2008 in Aleksić et al. (2015a) and then confirmed with more precise measurements from the 2009 campaign (Ahnen et al. 2017). The repeated occurrence of this variability pattern in 2012 demonstrates that this is a typical characteristic in the broadband emission of Mrk 501.

On the other hand, Furniss et al. (2015) show that, during the observing campaign in 2013, the multiband variability pattern was somewhat different, with the variability at X-rays being similar to that at VHE, thereby showing that somewhat different dynamical processes occurred in Mrk 501 during that year.

It is worth comparing this multiyear variability pattern of Mrk 501 with that from the other archetypical TeV blazar, Mrk 421. During the multi-instrument campaigns from 2009, 2010 and 2013, as reported in Aleksić et al. (2015b,c) and Baloković et al. (2016), Mrk 421 showed a double-peak 
structure in the plot of $F_{\text {var }}$ against energy, where the largest variability occurs in X-rays and VHE (instead of a broad increase with energy, with the variability at VHE being much larger than that at X-rays). These observations show a fundamentally different behavior when compared to that of Mrk 501.

During large VHE gamma-ray flaring activity, the X-ray and VHE gamma-ray emission of Mrk 501 have been found to be correlated. This occurred during the long and historical flare of 1997 (Pian et al. 1998; Gliozzi et al. 2006), and the large few-days-long flare observed in 2013 (Furniss et al. 2015). During non-flaring activity, a positive X-ray/VHE correlation was reported at the 99\% confidence level (Aleksić et al. 2015a). On the other hand, using the measurements from the 4.5-month-long 2009 campaign, where significant variability was observed in both X-ray and VHE bands, the emission from these two bands was found to be uncorrelated (Ahnen et al. 2017). Using the data collected during the 2012 campaign, with many more observations than in 2009 , one also finds only marginal correlation between the X-ray emission and the VHE gamma-ray emission. This is an interesting result because, under the most simplistic and widely accepted theoretical scenarios, the X-ray emission and the VHE gamma-ray emission are produced by the same population of high-energy particles (electrons and positrons). We note also that the situation for Mrk 421 is radically different from that of Mrk 501. The various multi-instrument campaigns performed on Mrk 421 always show a clear and positive correlation between the X-ray emission and the VHE gamma-ray emission, during both high and low source activity (Fossati et al. 2008; Acciari et al. 2011b; Aleksić et al. 2015c,b; Ahnen et al. 2016; Baloković et al. 2016).

Ahnen et al. (2017) put forward two scenarios to explain the measured multiband variability and correlations seen in the emission of Mrk 501: a) the high-energy electrons that are responsible for a large part of the $\mathrm{TeV}$ emission do not dominate the $\mathrm{keV}$ emission; b) there is an additional (and very variable) component contributing to the $\mathrm{TeV}$ emission, such as external inverseCompton. In this manuscript, we use the results from our one-zone SSC modeling of the 17 broadband SEDs from 2012 to probe the first scenario. We compared the one-zone SSC model fluxes at $0.5 \mathrm{keV}, 5 \mathrm{keV}$ and $50 \mathrm{keV}$ with the one-zone SSC model fluxes at $1 \mathrm{TeV}$, which are, by construction of the theoretical model, produced by the same population of electrons. The results are depicted in Fig. 8, and the correlations obtained are reported in Table 4. The X-ray vs VHE gamma-ray correlation as a function of the X-ray energy is: $3.3 \sigma$ for $0.5 \mathrm{keV}, 4.8 \sigma$ for $5 \mathrm{keV}$, and $3.8 \sigma$ for $50 \mathrm{keV}$. As reported in Sect. 6 (see Table 2), the June 9 flare (MJD 56087) is not properly described by the onezone SSC scenario used here. If we remove the results derived with the SSC model for this large flare, hence providing a more reliable description of the typical behavior of Mrk 501 during the campaign in 2012, the significance of the X-ray/VHE correlation is $1.7 \sigma$ for $0.5 \mathrm{keV}, 2.7 \sigma$ for $5 \mathrm{keV}$, and $3.4 \sigma$ for $50 \mathrm{keV}$. Therefore, the one-zone SSC model provides X-ray/VHE correlations at a level that are consistent with correlations obtained with the measured X-ray and VHE gamma-ray fluxes reported in Table 1 (Ahnen et al. 2017). This shows that an additional highenergy component (e.g. external inverse-Compton) is not necessary to explain the variability and correlation patterns observed in Mrk 501.

This exercise also shows the importance of sampling with accuracy a large portion of the electromagnetic spectrum.

In particular, sensitivity in the $50-100 \mathrm{keV}$ range comparable to that currently provided by MAGIC and VERITAS in the 0.1$1 \mathrm{TeV}$ range would greatly increase the potential for studying flux
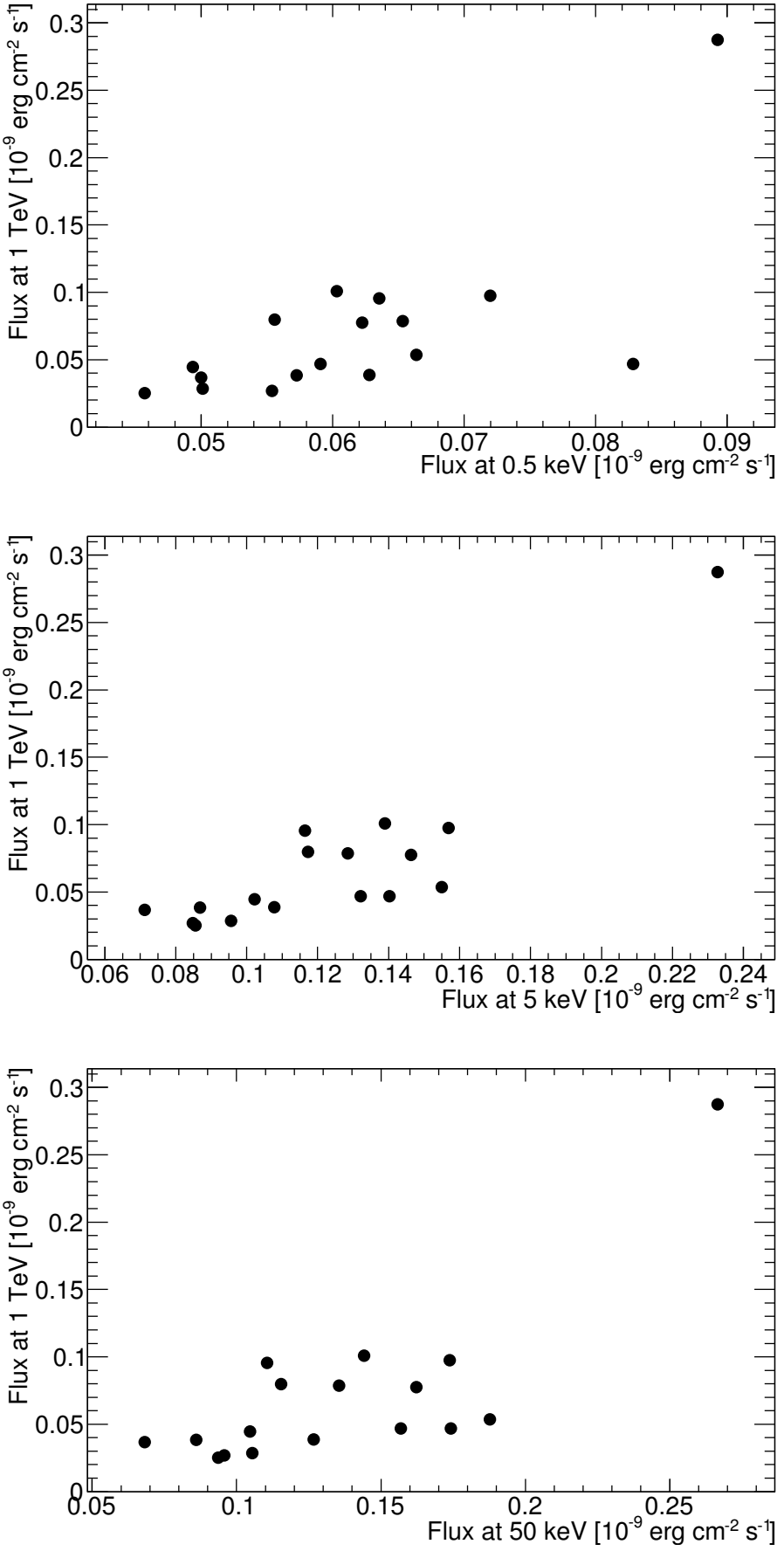

Fig. 8. Flux-flux plots derived from the one-zone SSC model used to fit 17 broadband SEDs (see Sect. 6). The SSC model flux at $1 \mathrm{TeV}$ is compared to the SSC model flux at $0.5 \mathrm{keV}$ (top panel), $5 \mathrm{keV}$ (middle panel) and $50 \mathrm{keV}$ (bottom panel). The data point with the highest X-ray and VHE activity corresponds to the one-zone SSC model for the June 9 flare (MJD 56087).

variability and interband correlations. The main differences in the multiband variability and correlation patterns with Mrk 421 may be related to the fact that, for Mrk 421, the electrons dominating the emission of the $\sim 1 \mathrm{TeV}$ photons also dominate the emission at $\sim 1 \mathrm{keV}$ (the peak of the synchrotron spectrum). This is the energy region sampled with $S$ wift/XRT with exquisite accuracy and extensive temporal coverage.

Recent and future publications devoted to multiwavelength campaigns on blazars will continue to benefit from a 
Table 4. Correlations derived for several combinations of X-ray bands and VHE flux above $1 \mathrm{TeV}$ using the data (upper part) and the one-zone SSC theoretical model used to describe 17 broadband SEDs.

\begin{tabular}{lr}
\hline \hline & $\begin{array}{r}\text { Pearson correlation } \\
\text { coefficient }(\sigma)\end{array}$ \\
\hline Data: 0.3-2 keV vs >1 TeV & $0.78_{-0.15}^{+0.10}(3.9)$ \\
Excluding June 9 flare: & $0.39_{-0.29}^{+0.23}(1.6)$ \\
Data: 2-10 keV vs >1 TeV & $0.88_{-0.10}^{+0.06}(4.9)$ \\
Excluding June 9 flare: & $0.59_{-0.24}^{+0.16}(2.5)$ \\
\hline Model: 0.5 keV vs 1 TeV & $0.71_{-0.16}^{+0.11}(3.3)$ \\
Excluding June 9 flare: & $0.44_{-0.25}^{+0.19}(1.7)$ \\
Model: 5.0 keV vs 1 TeV & $0.87_{-0.09}^{+0.06}(4.8)$ \\
Excluding June 9 flare: & $0.63_{-0.20}^{+0.14}(2.7)$ \\
Model: 50.0 keV vs 1 TeV & $0.77_{-0.13}^{+0.09}(3.8)$ \\
Excluding June 9 flare: & $0.73_{-0.16}^{+0.11}(3.4)$ \\
\hline
\end{tabular}

Notes. See text for further details.

new generation of X-ray telescopes, including $N U S T A R^{12}$ and Astrosat $^{13}$, which operate at $3-79 \mathrm{keV}$ and 2-80 keV respectively. NUSTAR represents a significant improvement (by a factor of 100 in sensitivity) over coded-mask instruments like Swift/BAT, and hence provides a much better view into the hard X-ray emission of blazars.

\section{Summary and conclusion}

We have presented the results from the 2012 Mrk 501 multiwavelength campaign. An excellent set of data was taken using more than 25 instruments over the period covering March to June 2012. The source flux was observed to vary between 0.5 and $4.9 \mathrm{CU}$ above $1 \mathrm{TeV}$, with an average flux of $\sim 1 \mathrm{CU}$. The highest VHE gamma-ray flux was observed on a single night, June 9 . This outburst was also observed in the X-ray band by the $S$ wift/XRT instrument.

The fractional variability was seen to increase as a function of energy and peak in the VHE regime. This is similar to what has been seen in other multiwavelength campaigns targeting Mrk 501, but different to the behavior of Mrk 421 which peaks at X-ray energies. This remains the case even when the flare information is removed or when we consider only data taken simultaneously in the X-ray and VHE bands, thereby underlining the difference already observed between these two sources.

Investigating possible correlations between X-ray and VHE bands, in two energy ranges each, a maximum Pearson correlation coefficient of $4.9 \sigma$ was observed for energies above $2 \mathrm{keV}$ and $1 \mathrm{TeV}$. The significance of this correlation drops to $2.5 \sigma$ when the flare day is excluded. A further search for correlation using a discrete correlation function found no evidence for a time lag between $\mathrm{X}$-rays and VHE gamma rays.

Interestingly, the X-ray and VHE power-law index corresponded to an extremely hard spectrum during the entire threemonth period. The VHE spectral index above $0.2 \mathrm{TeV}$, as observed by both MAGIC and VERITAS, was around $\sim 2$, compared to the typical power-law index of 2.5 (Acciari et al. 2011a; Abdo et al. 2011; Aleksić et al. 2015a). The source did not show the previously observed harder-when-brighter behavior at VHE energies. In the X-ray domain, Mrk 501 showed a hardening of the spectral

\footnotetext{
12 http://www.nustar.caltech.edu

13 http://astrosat.iucaa.in
}

shape with increasing X-ray flux, but the X-ray power-law spectral index was always less than 2 (for both low and high activity). Therefore, the synchrotron peak was located above $5 \mathrm{keV}$ and the inverse-Compton peak above $0.5 \mathrm{TeV}$, making Mrk 501 an extreme HBL during the entire observing period. This suggests that being an EHBL is a temporary state of the source, instead of an instrinsic characteristic.

We were able to form 17 SEDs, where the time difference between X-ray and VHE data taking was less than four hours. The X-ray and VHE data were modeled using a one-zone SSC model; however, the model underestimated the amount of optical and UV radiation required to fit the observed light curves. During 2012 the optical light curve was observed to be at a 10-year low and we assume that this component comes from a different region. The emission at $\mathrm{GeV}$ is systematically (within $1-2 \sigma$ ) above the 17 SSC model curves, showing a data-model difference of $\sim 3 \sigma$, which may be interpreted as a hint for the existence of an additional contribution at $\mathrm{GeV}$ energies, and is not considered in the current theoretical scenario. A two-zone model was also used in order to model the flare day of June 9 (MJD 56087). The two-zone SSC scenario improved the data-model agreement with respect to the one-zone SSC model and theoretical assumptions, although it still does not describe the broadband data well.

Despite the caveats mentioned above, the one-zone SSC framework provides a reasonable description of the segments of the SED where most energy is emitted, and where most of the variability occurs. The direct relation between the electron energy density and the gamma-ray activity shows that most of the variability can be explained by injection of high-energy electrons. The very hard $\operatorname{EED}\left(p_{1}<2\right)$ obtained in our fits, together with the trend of lower magnetic field strength $B$ for higher electron energy densities $U_{\mathrm{e}}$, suggests that magnetic reconnection plays a dominant role in the acceleration of the particles. However, we cannot exclude scenarios that incorporate other mechanisms for acceleration of the radiating (high-energy) particle population, such as efficient shock drift acceleration, or second-order Fermi acceleration if the electrons can be effectively trapped in regions of strong turbulence.

Acknowledgements. The MAGIC collaboration would like to thank the Instituto de Astrofísica de Canarias for the excellent working conditions at the Observatorio del Roque de los Muchachos in La Palma. The financial support of the German BMBF and MPG, the Italian INFN and INAF, the Swiss National Fund SNF, the ERDF under the Spanish MINECO (FPA2015-69818-P, FPA201236668, FPA2015-68378-P, FPA2015-69210-C6-2-R, FPA2015-69210-C6-4-R, FPA2015-69210-C6-6-R, AYA2015-71042-P, AYA2016-76012-C3-1-P, ESP2015-71662-C2-2-P, CSD2009-00064), and the Japanese JSPS and MEXT is gratefully acknowledged. This work was also supported by the Spanish Centro de Excelencia "Severo Ochoa" SEV-2012-0234 and SEV-2015-0548, and Unidad de Excelencia "María de Maeztu" MDM-2014-0369, by the Croatian Science Foundation (HrZZ) Project 09/176 and the University of Rijeka Project 13.12.1.3.02, by the DFG Collaborative Research Centers SFB823/C4 and SFB876/C3, the Polish National Research Centre grant UMO-2016/22/M/ST9/00382 and by the Brazilian MCTIC, CNPq and FAPERJ. VERITAS is supported by grants from the U.S. Department of Energy Office of Science, the U.S. National Science Foundation and the Smithsonian Institution, and by NSERC in Canada. We acknowledge the excellent work of the technical support staff at the Fred Lawrence Whipple Observatory and at the collaborating institutions in the construction and operation of the instrument. The Fermi-LAT Collaboration acknowledges generous ongoing support from a number of agencies and institutes that have supported both the development and the operation of the LAT as well as scientific data analysis. These include the National Aeronautics and Space Administration and the Department of Energy in the United States, the Commissariat à l'Energie Atomique and the Centre National de la Recherche Scientifique/Institut National de Physique Nucléaire et de Physique des Particules in France, the Agenzia Spaziale Italiana and the Istituto Nazionale di Fisica Nucleare in Italy, the Ministry of Education, Culture, Sports, Science and Technology (MEXT), High Energy Accelerator Research Organization (KEK) and Japan Aerospace 
Exploration Agency (JAXA) in Japan, and the K. A. Wallenberg Foundation, the Swedish Research Council and the Swedish National Space Board in Sweden. Additional support for science analysis during the operations phase is gratefully acknowledged from the Istituto Nazionale di Astrofisica in Italy and the Centre National d'Études Spatiales in France. This work performed in part under DOE Contract DE-AC02-76SF00515. The St.Petersburg University team acknowledges support from Russian Science Foundation grant 17-12-01029. The Abastumani team acknowledges financial support by the by Shota Rustaveli NSF under contract FR/577/6-320/13. This research was partially supported by the Bulgarian National Science Fund of the Ministry of Education and Science under grants DN 08-1/2016 and DN 18-13/2017. The Skinakas Observatory is a collaborative project of the University of Crete, the Foundation for Research and Technology -Hellas, and the Max-Planck-Institut für Extraterrestrische Physik. We would like to thank the American Association of Variable Star Observers (AAVSO) for making some of the observations used in this study. M.I.C acknowledges financial support by PRIN-SKA-CTA-INAF 2016. This article is partly based on observations made with the IAC80 telescope operated on the island of Tenerife by the Instituto de Astrofisica de Canarias in the Spanish Observatorio del Teide. The Liverpool Telescope is operated on the island of La Palma by Liverpool John Moores University in the Spanish Observatorio del Roque de los Muchachos of the Instituto de Astrofisica de Canarias with financial support from the UK Science and Technology Facilities Council. This work is partly based upon observations carried out at the Observatorio Astronómico Nacional on the Sierra San Pedro Mártir (OAN-SPM), Baja California, Mexico. The Steward Observatory spectropolarimetric monitoring program is supported by Fermi Guest Investigator grants NNX09AU10G and NNX12AO93G. The OVRO 40-m monitoring program is supported in part by NASA grants NNX08AW31G, NNX11A043G and NNX14AQ89G, and NSF grants AST-0808050 and AST-1109911. This publication makes use of data obtained at Metsähovi Radio Observatory, operated by Aalto University, Finland.

\section{References}

Abdo, A. A., Ackermann, M., Ajello, M., et al. 2011, ApJ, 727, 129 Acciari, V. A., Arlen, T., Aune, T., et al. 2011a, ApJ, 729, 2 Acciari, V. A., Aliu, E., \& Arlen, T. 2011b, ApJ, 738, 25

Acero, F., Ackermann, M., Ajello, M., et al. 2015, ApJS, 218, 23 Ackermann, M., Ajello, M., Allafort, A., et al. 2011, ApJ, 741, 30 Aharonian, F. A. 2000, Nature, 5, 377

Aharonian, F. A., Akhperjanian, A. G., Barrio, J. A., et al. 1999, A\&A, 349, 11

Aharonian, F., Akhperjanian, A. G., Barresde-Almeida, U., et al. 2007a, A\&A, 473, L25

Aharonian, F., Akhperjanian, A. G., Barresde-Almeida, U., et al. 2007b, A\&A, 475, L9

Ahnen, M. L., Ansoldi, S., Antonelli, L. A., et al. 2016, A\&A, 593, A91 Ahnen, M. L., Ansoldi, S., Antonelli, L. A., et al. 2017, A\&A, 603, A31 Albert, J., Aliu, E., Anderhub, H., et al. 2007, ApJ, 669, 862

Aleksić, J., Ansoldi, S., Antonelli, L. A., et al. 2014, A\&A, 572, A121 Aleksić, J., Ansoldi, S., Antonelli, L. A., et al. 2015a, A\&A, 573, A50 Aleksić, J., Ansoldi, S., Antonelli, L. A., et al. 2015b, A\&A, 576, A126 Aleksić, J., Ansoldi, S., Antonelli, L. A., et al. 2015c, A\&A, 578, A22 Aleksić, J., Ansoldi, S., Antonelli, L. A., et al. 2016, Astropart. Phys., 72, 76 Aliu, E., Archambault, S., Arlen, T., et al. 2014, ApJ, 782, 13 Aliu, E., Archambault, S., Archer, A., et al. 2016, A\&A, 594, A76 Anderhub, H., Backes, M., Biland, A., et al. 2013, J. Instrum., 8, 6008P Arnaud, K. A. 1996, in ASP Conf. Ser., eds. G. H. Jacoby, \& J. Barnes, 101, 17 Asano, K., Takahara, F., Kusunose, M., Toma, K., \& Kakuwa, J. 2014, ApJ, 780,64

Atwood, W. B., Abdo, A. A., Ackermann, M., et al. 2009, ApJ, 697, 1071 Baloković, M., Paneque, D., Madejski, G., et al. 2016, ApJ, 819, 156 Baring, M. G., Böttcher, M., \& Summerlin, E. J. 2017, MNRAS, 464, 4875 Bonnoli, G., Tavecchio, F., Ghisellini, G., \& Sbarrato, T. 2015, MNRAS, 451, 611

Breeveld, A. A., Landsman, W., \& Holland, S. T. 2011, in AIP Conf. Ser., eds. J. L. Racusin, \& N. Gehrels, 1358, 373

Bretz, T., Biland, A., \& Buß, J. 2014, ArXiv e-prints [arXiv:1407. 1988]

Burrows, D. N., Hill, J. E., Nousek, J. A., et al. 2005, Space Sci. Rev., 120, 165 Catanese, M., Bradbury, S. M., Breslin, A. C., et al. 1997, ApJ, 487, L143 Cerruti, M. 2013, ArXiv e-prints [arXiv: 1307.8091]

Cerutti, B., Uzdensky, D. A., \& Begelman, M. C. 2012a, ApJ, 746, 148

Cerutti, B., Werner, G. R., Uzdensky, D. A., \& Begelman, M. C. 2012b, ApJ, 754, L33

Cerruti, M., Zech, A., Boisson, C., \& Inoue, S. 2015, MNRAS, 448, 910

Chen, X., Pohl, M., \& Böttcher, M. 2015, MNRAS, 447, 530

Cologna, G., Mohamed, M., \& Wagner, S.J. 2015, ArXiv e-prints [arXiv: 1509.04470]
Costamante, L. 2013, Int. J. Mod. Phys. D, 22, 1330025

Costamante, L., Ghisellini, G., Giommi, P., et al. 2001, A\&A, 371, 512

D. B. Kieda for the VERITAS Collaboration 2013, ArXiv e-prints [arXiv: 1308.4849]

de Angelis, A., Roncadelli, M., \& Mansutti, O. 2007, Phys. Rev. D, 76, 121301 de Angelis, A., Galanti, G., \& Roncadelli, M. 2011, Phys. Rev. D, 84, 105030 Dermer, C. D., Schlickeiser, R., \& Mastichiadis, A. 1992, A\&A, 256, L27

DjannatiAtai, A., Piron, F., Barrau, A., et al. 1999, A\&A, 350, 17

Domínguez, A., \& Ajello, M. 2015, ApJ, 813, L34

Domínguez, A., Primack, J. R., Rosario, D. J., et al. 2011, MNRAS, 410, 2556 Dorner, D., Biland, A., \& Bretz, T. 2013, ArXiv e-prints [arXiv:1311.0478]

Dorner, D., Ahnen, M.L., Bergmann, M., et al. 2015, ArXiv e-prints [arXiv:1502.02582]

Dwek, E., \& Krennrich, F. 2013, Astropart. Phys., 43, 112

Edelson, R. A., \& Krolik, J. H. 1988, ApJ, 333, 646

Edwards, P. G., \& Piner, B. G. 2002, ApJ, 579, L67

Essey, W., \& Kusenko, A. 2010, Astropart. Phys., 33, 81

Fallah Ramazani, V., 2017, 7th International Fermi Symposium

Finke, J. D., Reyes, L. C., Georganopoulos, M., et al. 2015, ApJ, 814, 20

Fitzpatrick, E. L. 1999, PASP, 111, 63

Fossati, G., Buckley, J. H., Bond, I. H., et al. 2008, ApJ, 677, 906

Franceschini, A., Rodighiero, G., \& Vaccari, M. 2008, A\&A, 487, 837

Furniss, A., Noda, K., Boggs, S., et al. 2015, ApJ, 812, 65

Gehrels, N., Chincarini, G., Giommi, P., et al. 2004, ApJ, 611, 1005

Giannios, D., Uzdensky, D. A., \& Begelman, M. C. 2010, MNRAS, 402, 1649

Gilmore, R. C., Somerville, R. S., Primack, J. R., \& Domínguez, A. 2012, MNRAS, 422, 3189

Gliozzi, M., Sambruna, R. M., Jung, I., et al. 2006, ApJ, 646, 61

Holder, J., Acciari, V. A., \& Aliu, E. 2008, in AIP Conf. Ser., eds. F. A. Aharonian,

W. Hofmann, \& F. Rieger, 1085, 657

Jermak, H., Steele, I. A., Lindfors, E., et al. 2016, MNRAS, 462, 4267

Jorstad, S. G., Marscher, A. P., Larionov, V. M., et al. 2010, ApJ, 715, 362

Kalberla, P. M. W., Burton, W. B., Hartmann, D., et al. 2005, A\&A, 440, 775

Krawczynski, H., Hughes, S. B., Horan, D., et al. 2004, ApJ, 601, 151

Krimm, H. A., Holland, S. T., Corbet, R. H. D., et al. 2013, ApJS, 209, 14

Larionov, V. M., Jorstad, S. G., Marscher, A. P., et al. 2008, A\&A, 492, 389

Lefa, E., Rieger, F. M., \& Aharonian, F. 2011, ApJ, 740, 64

Lister, M. L., Aller, H. D., Aller, M. F., et al. 2009, AJ, 137, 3718

Madhavan, A. 2013, PhD Thesis, Iowa State Univ.

Mannheim, K. 1993, A\&A, 269, 67

Maraschi, L., Ghisellini, G., \& Celotti, A. 1992, ApJ, 397, L5

Markaryan, B. E., \& Lipovetskii, V. A. 1972, Astrophysics, 8, 89

Markwardt, C. B., Tueller, J., Skinner, G. K., et al. 2005, ApJ, 633, L77

Marscher, A. P., \& Gear, W. K. 1985, ApJ, 298, 114

Mattox, J. R., Bertsch, D. L., Chiang, J., et al. 1996, ApJ, 461, 396

Mirzoyan, R. HEGRA Collaboration 1998, New Astron. Rev., 42, 547

Neronov, A., \& Vovk, I. 2010, Science, 328, 73

Nilsson, K., Pasanen, M., Takalo, L. O., et al. 2007, A\&A, 475, 199

Paliya, V. S., Böttcher, M., Diltz, C., et al. 2015, ApJ, 811, 143

Pian, E., Vacanti, G., Tagliaferri, G., et al. 1998, ApJ, 492, L17

Piner, B. G., \& Edwards, P. G. 2004, ApJ, 600, 115

Piner, B. G., Pant, N., \& Edwards, P. G. 2010, ApJ, 723, 1150

Pohl, M., \& Schlickeiser, R. 2000, A\&A, 354, 395

Poole, T. S., Breeveld, A. A., Page, M. J., et al. 2008, MNRAS, 383, 627

Poutanen, J., Zdziarski, A. A., \& Ibragimov, A. 2008, MNRAS, 389, 1427

Press, W. H., Teukolsky, S. A., Vetterling, W. T., \& Flannery, B. P. 2002,

Numerical Recipes in $\mathrm{C}++$ : the Art of Scientific Computing

Quinn, J., Akerlof, C. W., Biller, S., et al. 1996, ApJ, 456, L83

Quinn, J., Bond, I. H., Boyle, P. J., et al. 1999, ApJ, 518, 693

Richards, J. L., Max-Moerbeck, W., Pavlidou, V., et al. 2011, ApJS, 194, 29

Roming, P. W. A., Kennedy, T. E., Mason, K. O., et al. 2005, Space Sci. Rev., 120,95

Samuelson, F. W., Biller, S. D., Bond, I. H., et al. 1998, ApJ, 501, L17

SánchezConde, M. A., Paneque, D., Bloom, E., Prada, F., \& Domínguez, A. 2009, Phys. Rev. D, 79, 123511

Saugé, L., \& Henri, G. 2004, ApJ, 616, 136

Schlafly, E. F., \& Finkbeiner, D. P. 2011, ApJ, 737, 103

Schlickeiser, R. 1985, A\&A, 143, 431

Shukla, A., Chitnis, V. R., Singh, B. B., et al. 2015, ApJ, 798, 2

Shukla, A., Mannheim, K., Chitnis, V. R., et al. 2016, ApJ, 832, 177

Sikora, M., Begelman, M. C., \& Rees, M. J. 1994, ApJ, 421, 153

Smith, P.S., Montiel, E., \& Rightley, S. 2009, ArXiv e-prints [arXiv: 0912 . 3621]

Stawarz, Ł., \& Petrosian, V. 2008, ApJ, 681, 1725

Summerlin, E. J., \& Baring, M. G. 2012, ApJ, 745, 63

Tammi, J., \& Duffy, P. 2009, MNRAS, 393, 1063

Tanaka, Y. T., Stawarz, Ł., Finke, J., et al. 2014, ApJ, 787, 155

Tavecchio, F., \& Ghisellini, G. 2016, MNRAS, 456, 2374

Tavecchio, F., Maraschi, L., Pian, E., et al. 2001, ApJ, 554, 725 
Tavecchio, F., Ghisellini, G., Ghirlanda, G., Costamante, L., \& Franceschini, A. 2009, MNRAS, 399, L59

Tavecchio, F., Ghisellini, G., Bonnoli, G., \& Foschini, L. 2011, MNRAS, 414, 3566

Teräsranta, H., Tornikoski, M., Mujunen, A., et al. 1998, A\&AS, 132, 305

Vaughan, S., Edelson, R., Warwick, R. S., \& Uttley, P. 2003, MNRAS, 345, 1271

Villata, M., Raiteri, C. M., Lanteri, L., Sobrito, G., \& Cavallone, M. 1998, A\&AS, 130,305

Villata, M., Raiteri, C. M., Larionov, V. M., et al. 2008, A\&A, 481, L79

Villata, M., Raiteri, C. M., Gurwell, M. A., et al. 2009, A\&A, 504, L9

Vovk, I., Taylor, A. M., Semikoz, D., \& Neronov, A. 2012, ApJ, 747, L14

Zanin, R., Carmona, E., \& Sitarek, J., et al. 2013, Proc of 33rd ICRC, Rio de Janeiro, Brazil, 773

Zhang, H., Chen, X., \& Böttcher, M. 2014, ApJ, 789, 66

Zhang, H., Chen, X., Böttcher, M., Guo, F., \& Li, H. 2015, ApJ, 804, 58

1 ETH Zurich, 8093 Zurich, Switzerland

2 Universitàdi Udine, and INFN Trieste, 33100 Udine, Italy

3 National Institute for Astrophysics (INAF), 00136 Rome, Italy

4 Università di Padova and INFN, 35131 Padova, Italy

5 Croatian MAGIC Consortium: University of Rijeka 51000 Rijeka; University of Split - FESB, 21000 Split; University of Zagreb - FER, 10000 Zagreb; University of Osijek, 31000 Osijek; Rudjer Boskovic Institute, 10000 Zagreb, Croatia

6 Saha Institute of Nuclear Physics, HBNI, 1/AF Bidhannagar, Salt Lake, Sector-1, Kolkata 700064, India

7 Max-Planck-Institut für Physik, 80805 München, Germany

8 Universidad Complutense, 28040 Madrid, Spain

9 Inst. de Astrofísica de Canarias; Universidad de La Laguna, Dpto. Astrofísica, 38206 La Laguna Tenerife, Spain

10 University of Łódź, Department of Astrophysics, 90236 Łódź, Poland

11 Deutsches Elektronen-Synchrotron (DESY), 15738 Zeuthen, Germany

12 Institut de Física d'Altes Energies (IFAE), The Barcelona Institute of Science and Technology (BIST), 08193 Bellaterra, Spain

13 Università di Siena, and INFN Pisa, 53100 Siena, Italy

14 Technische Universität Dortmund, 44221 Dortmund, Germany

15 Universität Würzburg, 97074 Würzburg, Germany

16 Finnish MAGIC Consortium: Tuorla Observatory and Finnish Centre of Astronomy with ESO (FINCA), University of Turku, Vaisalantie 20, FI-21500 Piikkiö; Astronomy Division, University of Oulu, 90014, Finland

17 Departament de Física, and CERES-IEEC, Universitat Autónoma de Barcelona, 08193 Bellaterra, Spain

18 Universitat de Barcelona, ICC, IEEC-UB, 08028 Barcelona, Spain

19 Japanese MAGIC Consortium: ICRR, The University of Tokyo $277-$ 8582 Chiba; Department of Physics, Kyoto University, 606-8502 Kyoto; Tokai University, 259-1292 Kanagawa; The University of Tokushima, 770-8502 Tokushima, Japan

20 Inst. for Nucl. Research and Nucl. Energy, Bulgarian Academy of Sciences, 1784 Sofia, Bulgaria

21 Università di Pisa, and INFN Pisa, 56126 Pisa, Italy

22 Centro Brasileiro de Pesquisas Físicas (CBPF), 22290-180 URCA Rio de Janeiro, Brasil

23 Humboldt University of Berlin, Institut für Physik, 12489 Berlin, Germany

24 Dipartimento di Fisica, Università di Trieste, 34127 Trieste, Italy

25 Port d'Informació Científica (PIC), 08193 Bellaterra, Spain

26 INAF-Trieste and Dept. of Physics \& Astronomy, University of Bologna, Italy

27 University of Geneva, ISDC Data Center for Astrophysics, Chemin dÉcogia 16, 1290 Versoix, Switzerland

28 RWTH Aachen University, III. Physikalisches Institut A, Aachen, Germany

29 Department of Physics, Washington University, St. Louis, MO 63130, USA

30 Fred Lawrence Whipple Observatory, Harvard-Smithsonian Center for Astrophysics, Amado, AZ 85645, USA

31 Department of Physics and Astronomy, University of California, Los Angeles, CA 90095, USA
32 Institute of Physics and Astronomy, University of Potsdam, 14476 Potsdam-Golm, Germany

33 Physics Department, California Polytechnic State University, San Luis Obispo, CA 94307, USA

34 Department of Physics and Astronomy, Purdue University, West Lafayette, IN 47907, USA

35 Department of Physics and Center for Astrophysics, Tsinghua University, Beijing 100084, PR China

36 Department of Astronomy and Astrophysics, 525 Davey Lab, Pennsylvania State University, University Park, PA 16802, USA

37 Physics Department, McGill University, Montreal, QC H3A 2T8, Canada

38 School of Physics, National University of Ireland Galway, University Road, Galway, Ireland

39 Santa Cruz Institute for Particle Physics and Department of Physics, University of California, Santa Cruz, CA 95064, USA

40 Department of Physics and Astronomy and the Bartol Research Institute, University of Delaware, Newark, DE 19716, USA

41 Physics Department, Columbia University, New York, NY 10027, USA

42 Department of Physics and Astronomy, University of Iowa, Van Allen Hall, Iowa City, IA 52242, USA

43 Department of Physics and Astronomy, University of Utah, Salt Lake City, UT 84112, USA

44 Department of Physics and Astronomy, DePauw University, Greencastle, IN 46135-0037, USA

45 Department of Physics and Astronomy, Iowa State University, Ames, IA 50011, USA

46 Department of Physics and Astronomy, Barnard College, Columbia University, NY 10027, USA

47 School of Physics, University College Dublin, Belfield, Dublin 4, Ireland

48 School of Physics and Center for Relativistic Astrophysics, Georgia Institute of Technology, 837 State Street NW, Atlanta, GA 303320430, USA

49 Enrico Fermi Institute, University of Chicago, Chicago, IL 60637, USA

50 Instituto de Astronomía y Física del Espacio (IAFE, CONICETUBA), CC 67 -Suc. 28 C1428ZAA, Ciudad Autónoma de Buenos Aires, Argentina

51 Department of Physical Sciences, Cork Institute of Technology, Bishopstown, Cork, Ireland

52 School of Physics and Astronomy, University of Minnesota, Minneapolis, MN 55455, USA

53 Department of Physics and Astronomy, University of Alabama, Tuscaloosa, AL 35487, USA

${ }^{54}$ Fred Lawrence Whipple Observatory, Harvard-Smithsonian Center for Astrophysics, Amado, AZ 85645, USA

55 Space Science Data Center - ASI, via del Politecnico, s.n.c., 00133 Roma, Italy

56 INAF, Osservatorio Astronomicodi Roma, via di Frascati 33, 00040 Monteporzio, Italy

57 INAF, Osservatorio Astrofisico di Torino, 10025 Pino Torinese, Italy

58 Astronomical Institute, St. Petersburg State University, Universitetskij Pr. 28, Petrodvorets, 198504 St. Petersburg, Russia

59 Pulkovo Observatory, St.-Petersburg, Russia

60 Department of Physics and Institute for Theoretical and Computational Physics (ITCP), University of Crete, 71003 Heraklion, Greece

${ }^{61}$ Foundation for Research and Technology - Hellas, IESL, Voutes, 7110 Heraklion, Greece

62 Abastumani Observatory, Mt. Kanobili, 0301 Abastumani, Georgia

63 Engelhardt Astronomical Observatory, Kazan Federal University, Tatarstan, Russia

${ }^{64}$ Xinjiang Astronomical Observatory, Chinese Academy of Sciences, Urumqi 830011, PR China

65 Institute of Astronomy and National Astronomical Observatory, Bulgarian Academy of Sciences, 72 Tsarigradsko shosse Blvd., 1784 Sofia, Bulgaria

66 Department of Physics, University of Colorado Denver, Denver, Colorado, CO 80217-3364, USA 
${ }^{67}$ Department of Physics and Astronomy, Brigham Young University, Provo, UT 84602, USA

68 Graduate Institute of Astronomy, National Central University, 300 Zhongda Road, Zhongli 32001, Taiwan

69 European Southern Observatory, Karl-Schwarzschild-Str. 2, 85748 Garching, Germany

70 Astrophysics Research Institute, Liverpool John Moores University, IC2, 146 Brownlow Hill, Liverpool L3 5RF, UK

71 Instituto de Astronomía, Universidad Nacional Autónoma de México, Apdo. Postal 70-264, 04510 Cd. de México, Mexico

72 Instituto de Astronomía, Universidad Nacional Autónoma de México, Apdo.Postal 810, 22800 Ensenada, B.C., Mexico

73 Steward Observatory, University of Arizona, Tucson, AZ 85721, USA

74 Universidad de Chile, Departamento de Astronomía, Camino El Observatorio 1515, Las Condes, Santiago, Chile
75 Cahill Centre for Astronomy and Astrophysics, California Institute of Technology, Pasadena, CA 91125, USA

76 Tuorla Observatory, Department of Physics and Astronomy, University of Turku, 20014 Turku, Finland

77 Aalto University Metsähovi Radio Observatory, Metsähovintie 114, 02540 Kylmälä, Finland

78 Aalto University Department of Electronics and Nanoengineering, PO BOX 15500, 00076 AALTO, Finland

79 Department of Physics, University of Maryland Baltimore County, 1000 Hilltop Circle, Baltimore, MD 21250, USA

${ }^{80}$ NASA Goddard Space Flight Center, Code 663, Greenbelt, MD 20771, USA

81 Department of Physics and Astronomy - MS 108, Rice University, 6100 Main Street, Houston, TX 77251-1892, USA

82 Institute for Astrophysical Research, Boston University, 725 Commonwealth Avenue, Boston, MA 02215, USA 


\section{Appendix A: Normalization of the FACT excess rates using the MAGIC fluxes above $1 \mathrm{TeV}$}

FACT and MAGIC are located within $100 \mathrm{~m}$ of each other and therefore observe under exactly the same atmospheric conditions. The instrumentation used in these two telescopes is different (e.g. FACT uses SiPMs as light detectors, instead of PMTs), and the observation mode (stereo vs mono) and analysis chains are completely separate. It is interesting and useful therefore to compare the two experiments' light curves. Figure A.1 shows the MAGIC light curve above $1 \mathrm{TeV}$ superimposed with the FACT light curve. The scale is chosen so that for the night of the highest flux the points overlap. As FACT monitors Mrk 501 every possible night, the FACT light curve is more densely sampled than that of MAGIC. The right panel of Fig. A.1 shows that there is an excellent agreement between the MAGIC VHE fluxes above $1 \mathrm{TeV}$ and the excess rates measured with FACT. The flux-flux plot is fit to a first order polynomial resulting in a $\chi^{2} /$ d.o.f. of $10.4 / 10$, with a slope of $(8.51 \pm 0.61) \times 10^{-13} \mathrm{~cm}^{-2} \mathrm{~s}^{-1} \mathrm{hr}^{-1}$ and an offset of $(-0.0021 \pm 0.0021) \times 10^{-10} \mathrm{~cm}^{-2} \mathrm{~s}^{-1} \mathrm{~h}^{-1}$, which is consistent with zero, as expected. This function was then used to normalize the FACT excess rate to the fluxes (above $1 \mathrm{TeV}$ ) reported in the bottom panel of Fig. 1.
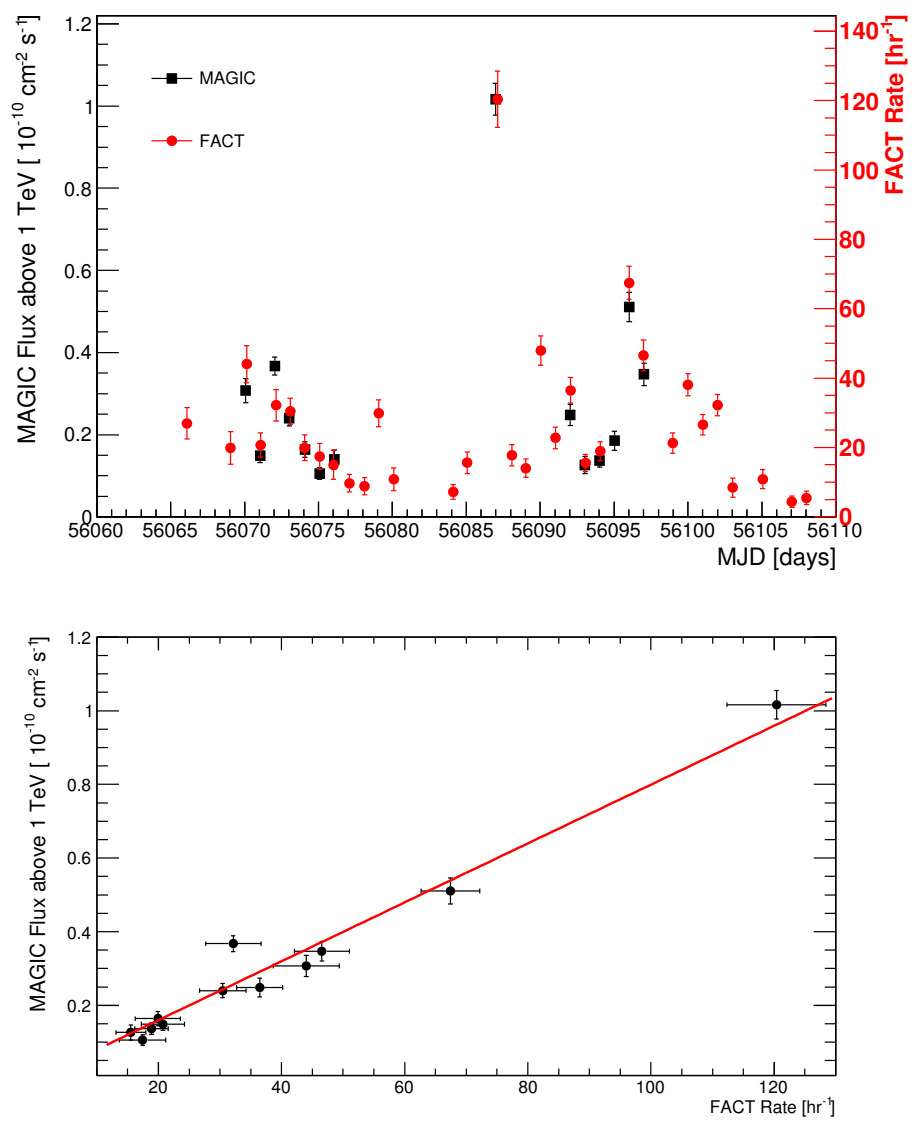

Fig. A.1. Top panel: superposition of the FACT excess rates and MAGIC light curve above $1 \mathrm{TeV}$. Bottom panel: correlation between the FACT excess rate and the MAGIC flux above $1 \mathrm{TeV}$ on the 12 days when data were taken by both instruments.

\section{Appendix B: Spectral fits to the gamma-ray and $\mathrm{X}$-ray spectra}

This section reports the spectral parameters resulting from the fits to the X-ray and gamma-ray spectra.

Table B.1. Parameters resulting from the fit with a power law $F(E)=$ $N_{0}(E / 0.5 \mathrm{TeV})^{-\Gamma}$ to the measured MAGIC spectra shown in Figs. 4-7.

\begin{tabular}{lccc}
\hline \hline MJD & $N_{0}\left(10^{-11} \mathrm{~s}^{-1} \mathrm{~cm}^{-2} \mathrm{TeV}^{-1}\right)$ & $\Gamma$ & $\chi^{2} /$ d.o.f. \\
\hline 56007 & $1.90 \pm 0.25$ & $1.85 \pm 0.25$ & $0.70 / 3$ \\
56032 & $2.39 \pm 0.23$ & $1.89 \pm 0.15$ & $1.49 / 3$ \\
56036 & $4.36 \pm 0.30$ & $1.88 \pm 0.08$ & $0.33 / 4$ \\
56040 & $2.46 \pm 0.23$ & $2.31 \pm 0.18$ & $1.36 / 3$ \\
56070 & $5.15 \pm 0.25$ & $1.94 \pm 0.08$ & $1.99 / 3$ \\
56071 & $2.85 \pm 0.18$ & $2.22 \pm 0.09$ & $6.76 / 5$ \\
56072 & $5.60 \pm 0.20$ & $2.02 \pm 0.04$ & $8.94 / 6$ \\
56073 & $4.73 \pm 0.23$ & $2.20 \pm 0.07$ & $18.37 / 6$ \\
56074 & $2.41 \pm 0.18$ & $2.01 \pm 0.09$ & $5.63 / 5$ \\
56075 & $1.83 \pm 0.15$ & $2.11 \pm 0.11$ & $5.49 / 5$ \\
56076 & $2.24 \pm 0.23$ & $2.20 \pm 0.15$ & $6.79 / 5$ \\
56087 & $15.43 \pm 0.31$ & $2.02 \pm 0.03$ & $96.26 / 6$ \\
56093 & $2.50 \pm 0.21$ & $2.15 \pm 0.12$ & $4.26 / 5$ \\
56094 & $2.60 \pm 0.17$ & $2.24 \pm 0.09$ & $3.09 / 5$ \\
56095 & $2.75 \pm 0.21$ & $2.05 \pm 0.10$ & $2.70 / 5$ \\
56096 & $7.15 \pm 0.30$ & $2.09 \pm 0.05$ & $12.94 / 6$ \\
56097 & $6.17 \pm 0.29$ & $2.20 \pm 0.07$ & $5.82 / 5$ \\
\hline
\end{tabular}

Notes. The fitted spectra were EBL corrected using Franceschini et al. (2008).

Table B.2. Parameters resulting from the fit with a power law $\mathrm{F}(\mathrm{E})=N_{0}(\mathrm{E} / 0.5 \mathrm{TeV})^{-\Gamma}$ to the measured VERITAS spectra shown in Figs. 4-6.

\begin{tabular}{lccc}
\hline \hline MJD & $N_{0}\left(10^{-11} \mathrm{~s}^{-1} \mathrm{~cm}^{-2} \mathrm{TeV}^{-1}\right)$ & $\Gamma$ & $\chi^{2} /$ d.o.f. \\
\hline 56007 & $1.08 \pm 0.09$ & $2.05 \pm 0.10$ & $10.73 / 6$ \\
56009 & $2.26 \pm 0.19$ & $1.99 \pm 0.09$ & $20.46 / 7$ \\
56015 & $1.82 \pm 0.17$ & $1.75 \pm 0.09$ & $6.80 / 6$ \\
56034 & $0.89 \pm 0.11$ & $1.59 \pm 0.21$ & $10.82 / 6$ \\
56038 & $0.58 \pm 0.09$ & $1.91 \pm 0.24$ & $9.01 / 5$ \\
56046 & $0.65 \pm 0.08$ & $1.80 \pm 0.18$ & $4.85 / 6$ \\
56050 & $0.70 \pm 0.08$ & $2.09 \pm 0.23$ & $5.39 / 6$ \\
56061 & $0.71 \pm 0.08$ & $2.19 \pm 0.19$ & $9.15 / 4$ \\
56066 & $1.12 \pm 0.09$ & $2.10 \pm 0.09$ & $1.74 / 6$ \\
56069 & $1.05 \pm 0.08$ & $2.02 \pm 0.13$ & $4.05 / 4$ \\
56073 & $2.27 \pm 0.12$ & $2.08 \pm 0.05$ & $10.22 / 5$ \\
56075 & $2.52 \pm 0.45$ & $2.12 \pm 0.22$ & $3.97 / 2$ \\
56076 & $1.35 \pm 0.14$ & $1.94 \pm 0.11$ & $1.73 / 3$ \\
56077 & $1.62 \pm 0.28$ & $1.23 \pm 0.26$ & $0.42 / 3$ \\
56090 & $2.08 \pm 0.40$ & $1.76 \pm 0.29$ & $12.39 / 4$ \\
56092 & $3.57 \pm 0.30$ & $2.48 \pm 0.19$ & $11.57 / 6$ \\
56093 & $1.46 \pm 0.35$ & $1.43 \pm 0.23$ & $3.39 / 2$ \\
56094 & $2.14 \pm 0.31$ & $1.81 \pm 0.29$ & $2.42 / 3$ \\
56095 & $2.06 \pm 0.30$ & $1.95 \pm 0.24$ & $2.22 / 4$ \\
56096 & $2.55 \pm 0.31$ & $1.54 \pm 0.26$ & $5.07 / 3$ \\
56097 & $1.76 \pm 0.23$ & $1.91 \pm 0.17$ & $8.97 / 3$ \\
56099 & $0.85 \pm 0.15$ & $1.66 \pm 0.20$ & $1.16 / 2$ \\
\hline
\end{tabular}

Notes. The fitted spectra were EBL corrected using Franceschini et al. (2008). 
Table B.3. Fermi-LAT flux and power-law index above $0.2 \mathrm{GeV}$ from the gamma-ray spectra shown in Figs. 5-7.

\begin{tabular}{cccc}
\hline \hline Observation (MJD) & Flux $\left(10^{-8} \mathrm{~cm}^{-2} \mathrm{~s}^{-1}\right)$ & $\Gamma$ & $\mathrm{TS}$ \\
\hline $56007.5-56010.5$ & $6.3 \pm 2.1$ & $1.7 \pm 0.2$ & 43 \\
$56013.5-56016.5$ & $7.3 \pm 2.4$ & $1.8 \pm 0.2$ & 61 \\
$56030.5-56033.5$ & $4.9 \pm 2.0$ & $1.9 \pm 0.3$ & 34 \\
$56032.5-56035.5$ & $3.6 \pm 1.9$ & $1.9 \pm 0.4$ & 19 \\
$56034.5-56037.5$ & $5.0 \pm 2.7$ & $2.4 \pm 0.6$ & 16 \\
$56036.5-56039.5$ & $2.2 \pm 1.2$ & $1.4 \pm 0.3$ & 33 \\
$56038.5-56041.5$ & $2.9 \pm 1.9$ & $1.6 \pm 0.3$ & 28 \\
$56044.5-56047.5$ & $8.4 \pm 2.7$ & $2.0 \pm 0.2$ & 52 \\
$56059.5-56062.5$ & $1.1 \pm 1.1$ & $1.8 \pm 0.7$ & 4 \\
$56064.5-56067.5$ & $7.6 \pm 2.7$ & $2.1 \pm 0.3$ & 40 \\
$56071.5-56074.5$ & $5.7 \pm 1.9$ & $1.7 \pm 0.2$ & 56 \\
$56074.5-56077.5$ & $2.3 \pm 1.3$ & $1.4 \pm 0.3$ & 24 \\
$56075.5-56078.5$ & $4.0 \pm 1.6$ & $1.5 \pm 0.2$ & 41 \\
$56086.5-56087.5$ & $12.0 \pm 5.0$ & $1.5 \pm 0.2$ & 59 \\
$56088.5-56091.5$ & $8.4 \pm 2.9$ & $1.7 \pm 0.2$ & 71 \\
$56092.5-56095.5$ & $8.1 \pm 2.5$ & $1.8 \pm 0.2$ & 65 \\
$56093.5-56096.5$ & $7.5 \pm 2.3$ & $1.7 \pm 0.2$ & 72 \\
\hline
\end{tabular}


M. L. Ahnen et al.: Extreme HBL behavior of Markarian 501 during 2012

Table B.4. Spectral models (log-parabola and power-law functions) fitted to the Swift-XRT data used in Figs. 4-7.

\begin{tabular}{|c|c|c|c|c|c|c|c|}
\hline $\begin{array}{l}\text { Start time } \\
\text { MJD }\end{array}$ & $\alpha$ & $\beta$ & $\chi^{2} /$ d.o.f. & $\begin{array}{c}0.3-2 \mathrm{keV} \\
\left(10^{-10} \mathrm{erg} \mathrm{cm}^{-2} \mathrm{~s}^{-1}\right)\end{array}$ & $\begin{array}{c}2-10 \mathrm{keV} \\
\left(10^{-10} \mathrm{erg} \mathrm{cm}^{-2} \mathrm{~s}^{-1}\right)\end{array}$ & $\alpha$ & $\chi^{2} /$ d.o.f. \\
\hline 55972.079 & $1.532 \pm 0.052$ & $0.356 \pm 0.094$ & $189 / 221$ & $1.545 \pm 0.024$ & $2.062 \pm 0.069$ & $1.687 \pm 0.028$ & $233 / 222$ \\
\hline 55977.296 & $1.624 \pm 0.053$ & $0.411 \pm 0.103$ & $226 / 205$ & $1.144 \pm 0.018$ & $1.255 \pm 0.046$ & $1.778 \pm 0.028$ & $277 / 206$ \\
\hline 55981.242 & $1.694 \pm 0.055$ & $0.282 \pm 0.107$ & $176 / 198$ & $1.041 \pm 0.019$ & $1.147 \pm 0.047$ & $1.809 \pm 0.033$ & $196 / 199$ \\
\hline 55985.247 & $1.757 \pm 0.052$ & $0.261 \pm 0.102$ & $191 / 191$ & $1.427 \pm 0.026$ & $1.443 \pm 0.054$ & $1.862 \pm 0.033$ & $210 / 192$ \\
\hline 55989.256 & $1.669 \pm 0.047$ & $0.237 \pm 0.093$ & $211 / 219$ & $1.346 \pm 0.021$ & $1.609 \pm 0.051$ & $1.761 \pm 0.028$ & $230 / 220$ \\
\hline 55994.206 & $1.712 \pm 0.046$ & $0.347 \pm 0.092$ & $190 / 222$ & $1.306 \pm 0.021$ & $1.316 \pm 0.044$ & $1.849 \pm 0.028$ & $233 / 223$ \\
\hline 56000.142 & $1.634 \pm 0.047$ & $0.402 \pm 0.091$ & $270 / 229$ & $1.650 \pm 0.023$ & $1.797 \pm 0.057$ & $1.804 \pm 0.026$ & $329 / 230$ \\
\hline 56005.166 & $1.638 \pm 0.055$ & $0.363 \pm 0.108$ & $208 / 195$ & $1.628 \pm 0.029$ & $1.822 \pm 0.074$ & $1.782 \pm 0.033$ & $243 / 196$ \\
\hline 56009.429 & $1.662 \pm 0.047$ & $0.267 \pm 0.072$ & $221 / 229$ & $1.374 \pm 0.021$ & $1.617 \pm 0.054$ & $1.776 \pm 0.027$ & $247 / 230$ \\
\hline 56011.450 & $1.519 \pm 0.049$ & $0.378 \pm 0.088$ & $264 / 245$ & $1.447 \pm 0.020$ & $1.930 \pm 0.054$ & $1.691 \pm 0.026$ & $320 / 246$ \\
\hline 56015.451 & $1.606 \pm 0.055$ & $0.321 \pm 0.095$ & $203 / 217$ & $1.408 \pm 0.023$ & $1.725 \pm 0.055$ & $1.758 \pm 0.029$ & $237 / 218$ \\
\hline 56017.316 & $1.685 \pm 0.051$ & $0.214 \pm 0.095$ & $204 / 220$ & $1.370 \pm 0.024$ & $1.629 \pm 0.057$ & $1.780 \pm 0.029$ & $219 / 221$ \\
\hline 56019.188 & $1.714 \pm 0.044$ & $0.250 \pm 0.087$ & $232 / 230$ & $1.257 \pm 0.020$ & $1.380 \pm 0.043$ & $1.812 \pm 0.027$ & $256 / 231$ \\
\hline 56019.457 & $1.623 \pm 0.051$ & $0.342 \pm 0.099$ & $177 / 202$ & $1.186 \pm 0.018$ & $1.387 \pm 0.053$ & $1.755 \pm 0.029$ & $214 / 203$ \\
\hline 56023.080 & $1.760 \pm 0.059$ & $0.260 \pm 0.116$ & $185 / 181$ & $1.095 \pm 0.024$ & $1.104 \pm 0.044$ & $1.866 \pm 0.034$ & $200 / 182$ \\
\hline 56027.548 & $1.767 \pm 0.057$ & $0.302 \pm 0.113$ & $198 / 187$ & $1.173 \pm 0.024$ & $1.125 \pm 0.044$ & $1.890 \pm 0.033$ & $220 / 188$ \\
\hline 56032.174 & $1.664 \pm 0.054$ & $0.297 \pm 0.100$ & $183 / 215$ & $1.330 \pm 0.025$ & $1.518 \pm 0.054$ & $1.796 \pm 0.030$ & $209 / 216$ \\
\hline 56034.249 & $1.607 \pm 0.054$ & $0.298 \pm 0.103$ & $219 / 190$ & $1.080 \pm 0.019$ & $1.350 \pm 0.053$ & $1.728 \pm 0.031$ & $244 / 191$ \\
\hline 56036.043 & $1.590 \pm 0.057$ & $0.305 \pm 0.104$ & $176 / 208$ & $1.229 \pm 0.021$ & $1.568 \pm 0.055$ & $1.727 \pm 0.031$ & $202 / 209$ \\
\hline 56038.376 & $1.677 \pm 0.058$ & $0.397 \pm 0.112$ & $186 / 189$ & $1.003 \pm 0.019$ & $1.024 \pm 0.040$ & $1.846 \pm 0.033$ & $223 / 190$ \\
\hline 56040.123 & $1.710 \pm 0.054$ & $0.313 \pm 0.109$ & $213 / 196$ & $1.169 \pm 0.022$ & $1.218 \pm 0.047$ & $1.836 \pm 0.033$ & $237 / 197$ \\
\hline 56042.395 & $1.795 \pm 0.061$ & $0.258 \pm 0.125$ & $162 / 167$ & $1.199 \pm 0.028$ & $1.144 \pm 0.051$ & $1.894 \pm 0.038$ & $174 / 168$ \\
\hline 56044.005 & $1.706 \pm 0.075$ & $0.326 \pm 0.135$ & $119 / 165$ & $1.100 \pm 0.025$ & $1.142 \pm 0.049$ & $1.857 \pm 0.039$ & $136 / 166$ \\
\hline 56046.412 & $1.691 \pm 0.057$ & $0.298 \pm 0.111$ & $197 / 193$ & $1.108 \pm 0.021$ & $1.208 \pm 0.048$ & $1.815 \pm 0.034$ & $218 / 194$ \\
\hline 56047.413 & $1.673 \pm 0.057$ & $0.265 \pm 0.099$ & $200 / 205$ & $1.128 \pm 0.021$ & $1.306 \pm 0.045$ & $1.793 \pm 0.031$ & $219 / 206$ \\
\hline 56048.148 & $1.574 \pm 0.057$ & $0.323 \pm 0.111$ & $195 / 196$ & $1.020 \pm 0.019$ & $1.312 \pm 0.053$ & $1.711 \pm 0.033$ & $220 / 197$ \\
\hline 56053.089 & $1.678 \pm 0.054$ & $0.296 \pm 0.107$ & $186 / 192$ & $1.053 \pm 0.019$ & $1.175 \pm 0.044$ & $1.796 \pm 0.033$ & $208 / 193$ \\
\hline 56054.947 & $1.732 \pm 0.059$ & $0.341 \pm 0.112$ & $167 / 185$ & $1.111 \pm 0.022$ & $1.090 \pm 0.044$ & $1.877 \pm 0.034$ & $193 / 186$ \\
\hline 56056.029 & $1.624 \pm 0.056$ & $0.321 \pm 0.085$ & $181 / 197$ & $1.067 \pm 0.019$ & $1.270 \pm 0.047$ & $1.764 \pm 0.032$ & $208 / 198$ \\
\hline 56059.358 & $1.712 \pm 0.054$ & $0.226 \pm 0.102$ & & $1.069 \pm 0.019$ & $1.203 \pm 0.043$ & $1.807 \pm 0.032$ & \\
\hline 56061.296 & $1.700 \pm 0.057$ & $0.289 \pm 0.107$ & $178 / 187$ & $1.115 \pm 0.022$ & $1.207 \pm 0.047$ & $1.825 \pm 0.034$ & 199/188 \\
\hline 56066.310 & $1.578 \pm 0.070$ & $0.509 \pm 0.144$ & $164 / 130$ & $1.645 \pm 0.035$ & $1.779 \pm 0.093$ & $1.770 \pm 0.039$ & $202 / 131$ \\
\hline 56073.333 & $1.576 \pm 0.047$ & $0.225 \pm 0.088$ & $224 / 236$ & $1.355 \pm 0.020$ & $1.908 \pm 0.060$ & $1.671 \pm 0.027$ & $243 / 237$ \\
\hline 56074.059 & $1.628 \pm 0.062$ & $0.168 \pm 0.113$ & $183 / 184$ & $1.330 \pm 0.028$ & $1.817 \pm 0.074$ & $1.704 \pm 0.035$ & $190 / 185$ \\
\hline 56076.021 & $1.565 \pm 0.050$ & $0.336 \pm 0.095$ & $189 / 224$ & $1.371 \pm 0.022$ & $1.771 \pm 0.057$ & $1.709 \pm 0.028$ & $226 / 225$ \\
\hline 56077.273 & $1.608 \pm 0.048$ & $0.274 \pm 0.088$ & $212 / 232$ & $1.451 \pm 0.024$ & $1.850 \pm 0.061$ & $1.729 \pm 0.027$ & $241 / 233$ \\
\hline 56078.208 & $1.565 \pm 0.065$ & $0.383 \pm 0.122$ & $172 / 166$ & $1.311 \pm 0.026$ & $1.619 \pm 0.068$ & $1.723 \pm 0.035$ & $202 / 167$ \\
\hline 56078.423 & $1.636 \pm 0.056$ & $0.236 \pm 0.100$ & $200 / 215$ & $1.316 \pm 0.024$ & $1.661 \pm 0.058$ & $1.745 \pm 0.031$ & $216 / 216$ \\
\hline 56086.973 & $1.538 \pm 0.051$ & $0.190 \pm 0.090$ & $249 / 241$ & $2.055 \pm 0.031$ & $3.192 \pm 0.103$ & $1.627 \pm 0.027$ & $262 / 242$ \\
\hline 56089.047 & $1.637 \pm 0.053$ & $0.339 \pm 0.101$ & $208 / 216$ & $2.187 \pm 0.037$ & $2.506 \pm 0.084$ & $1.786 \pm 0.030$ & $241 / 217$ \\
\hline 56090.049 & $1.542 \pm 0.045$ & $0.291 \pm 0.083$ & $229 / 255$ & $1.591 \pm 0.022$ & $2.225 \pm 0.063$ & $1.669 \pm 0.025$ & $265 / 256$ \\
\hline 56090.923 & $1.660 \pm 0.046$ & $0.327 \pm 0.088$ & $207 / 226$ & $1.404 \pm 0.022$ & $1.568 \pm 0.051$ & $1.790 \pm 0.027$ & $248 / 227$ \\
\hline 56093.988 & $1.704 \pm 0.086$ & $0.198 \pm 0.155$ & $105 / 105$ & $0.954 \pm 0.028$ & $1.117 \pm 0.061$ & $1.796 \pm 0.049$ & $110 / 106$ \\
\hline 56095.050 & $1.543 \pm 0.050$ & $0.321 \pm 0.095$ & $236 / 235$ & $1.532 \pm 0.022$ & $2.078 \pm 0.069$ & $1.679 \pm 0.028$ & $271 / 236$ \\
\hline 56101.280 & $1.528 \pm 0.056$ & $0.384 \pm 0.106$ & $246 / 214$ & $1.620 \pm 0.025$ & $2.120 \pm 0.075$ & $1.697 \pm 0.030$ & $285 / 215$ \\
\hline 56103.281 & $1.563 \pm 0.045$ & $0.273 \pm 0.082$ & $261 / 261$ & $1.555 \pm 0.023$ & $2.137 \pm 0.061$ & $1.689 \pm 0.025$ & $294 / 262$ \\
\hline 56110.359 & $1.555 \pm 0.077$ & $0.340 \pm 0.147$ & $108 / 122$ & $1.081 \pm 0.025$ & $1.411 \pm 0.069$ & $1.700 \pm 0.042$ & $124 / 123$ \\
\hline 56116.297 & $1.803 \pm 0.062$ & $0.110 \pm 0.119$ & $165 / 163$ & $1.040 \pm 0.025$ & $1.120 \pm 0.054$ & $1.849 \pm 0.038$ & $168 / 164$ \\
\hline 56131.468 & $1.538 \pm 0.057$ & $0.293 \pm 0.105$ & $171 / 198$ & $1.296 \pm 0.023$ & $1.820 \pm 0.068$ & $1.665 \pm 0.032$ & $194 / 199$ \\
\hline 56138.152 & $1.438 \pm 0.042$ & $0.179 \pm 0.072$ & $281 / 288$ & $1.807 \pm 0.023$ & $3.348 \pm 0.082$ & $1.524 \pm 0.023$ & $299 / 289$ \\
\hline 55959.895 & $1.562 \pm 0.047$ & $0.321 \pm 0.084$ & $240 / 247$ & $1.494 \pm 0.021$ & $1.964 \pm 0.057$ & $1.709 \pm 0.025$ & $283 / 248$ \\
\hline 55966.582 & $1.652 \pm 0.054$ & $0.280 \pm 0.103$ & $161 / 190$ & $1.501 \pm 0.028$ & $1.774 \pm 0.067$ & $1.764 \pm 0.032$ & $183 / 191$ \\
\hline
\end{tabular}

